\title{
O teorema da alternativa de Tits
}

\author{
Renan Campos Gutierrez
}


SERVIÇO DE PÓS-GRADUAÇÃO DO ICMC-USP

Data de Depósito:

Assinatura:

\title{
O teorema da alternativa de Tits
}

\author{
Renan Campos Gutierrez
}

Orientador: Prof. Dr. Daniel Levcovitz

Dissertação apresentada ao Instituto de Ciências Matemáticas e de Computação - ICMC-USP, como parte dos requisitos para obtenção do título de Mestre em Ciências - Matemática . VERSÃO REVISADA

USP - São Carlos

Julho de 2012 
Ficha catalográfica elaborada pela Biblioteca Prof. Achille Bassi e Seção Técnica de Informática, ICMC/USP, com os dados fornecidos pelo(a) autor(a)

\begin{tabular}{|c|c|}
\hline \multirow[t]{3}{*}{ G983t } & $\begin{array}{l}\text { Gutierrez, Renan Campos } \\
\quad \text { O Teorema da Alternativa de Tits / Renan Campos } \\
\text { Gutierrez; orientador Daniel Levcovitz. -- São } \\
\text { Carlos, } 2012 \text {. } \\
\quad 51 \mathrm{p} .\end{array}$ \\
\hline & $\begin{array}{l}\text { Dissertação (Mestrado - Programa de Pós-Graduação en } \\
\text { Matemática)-- Instituto de Ciências Matemáticas e } \\
\text { de Computação, Universidade de São Paulo, } 2012 \text {. }\end{array}$ \\
\hline & $\begin{array}{l}\text { 1. Teoria de grupos. 2. Grupos lineares. } 3 . \\
\text { Grupos livres. 4. Alternativa de Tits. I. Levcovitz, } \\
\text { Daniel, orient. II. Título. }\end{array}$ \\
\hline
\end{tabular}


À minha mãe Rosimeire e ao meu pai Renato. 



\section{Agradecimentos}

Primeiramente a Deus, por todo apoio espiritual e pela forte presença em minha vida.

Agradeço imensamente aos meus pais Renato Vita Gutierrez e Rosimeire Aparecida Campos, que não mediram esforços para que este grande sonho se tornasse realidade.

À minha irmã Reniely, pela confiança e atenção. Agradeço muito à minha falecida bisavó Maria Ovídeo, que ajudou em minha criação e me deu conselhos que serviram de base para o meu caráter e personalidade.

Aos meus avós maternos João Campos e Maria Aparecida Magri Campos, pela ajuda fundamental em todos os aspectos e incentivo.

Aos meus tios André Trevizan e Rosana Cláudia Campos Trevizan, pelo carinho e incentivo.

Aos amigos de sempre: Adelis, Ana Cláudia, Guilherme, Leandro Tavares, Lucas, Natália e Wellington, pela atenção, carinho e as boas risadas que me proporcionaram . Em especial agradeço ao meu grande amigo Wilson, por todo seu apoio.

À todos os novos colegas que fiz em São Carlos.

Agradeço aos meus professores e orientadores de iniciação científica do IBILCE - Unesp, e também à todos os meus colegas de graduação, em especial os que fiz quando fazia parte do Pet.

Ao meu professor e orientador de mestrado Daniel Levcovitz, pela ajuda na elaboração da dissertação, pela atenção, educação e por ter aceitado me orientar.

Aos meus professores do ICMC-USP.

À FAPESP, pelo suporte financeiro para a realização do curso de mestrado.

Enfim, agradeço também a todos cujos nomes não aparecem aqui, mas cuja amizade foram fundamentais para que eu pudesse trilhar este caminho. 



\section{Resumo}

Este projeto de mestrado tem por objetivo dar uma prova "elementar" do seguinte teorema de Tits, conhecido como Teorema da Alternativa de Tits: Seja G um grupo linear finitamente gerado sobre um corpo. Então $G$ é solúvel por finito ou G contém um grupo livre não cíclico. Este teorema, que foi provado por J. Tits em 1972 [4], foi considerado pelo matemático J.P. Serre como um dos mais importantes resultados de álgebra do século XX. Quando dizemos uma prova "elementar", não queremos absolutamente te dizer uma prova simples. Seguiremos a prova simplificada de John D. Dixon. 



\section{Abstract}

This master's project aims to give an "elementary" proof of the following theorem of Tits, known as the Alternative Tits Theorem: Let $\mathrm{G}$ be a finitely generated linear group over a field. Then either $\mathrm{G}$ is solvable by finite or $\mathrm{G}$ contains a noncyclic free subgroup. This theorem was proved by J. Tits in 1972 [4], was considered by the mathematician J.P. Serre, as one of the most important algebra results of the XX century. When we say an elementary proof, we absolutely not mean a simple proof. We will follow the simplified proof of John D. Dixon. 



\section{Sumário}

$\begin{array}{ll}\text { Introdução } & 1\end{array}$

$\begin{array}{lr}\text { Notações } & 2\end{array}$

1 Preliminares $\quad 4$

1.1 Topologia de Zariski $\ldots \ldots \ldots \ldots \ldots$

1.2 Representações lineares, grupos redutíveis, irredutíveis e completamente redutíveis 8

1.3 Alguns grupos lineares clássicos . . . . . . . . . . . . . . . . 10

1.4 Lema do Ping-Pong . . . . . . . . . . . . . . . . . . . . . . . . 10

2 Valores absolutos em corpos $\quad 13$

2.1 Valores absolutos em um corpo . . . . . . . . . . . . . . . . 13

2.2 Topologia induzida por um valor absoluto . . . . . . . . . . . . . . 15

2.3 Completamento: os corpos p-ádicos $\mathbb{Q}_{p} \ldots \ldots \ldots \ldots$

2.4 Extensões finitas de corpos completos . . . . . . . . . . . . . . . . 18

2.5 Corpos locais . . . . . . . . . . . . . . . . . 23

3 Prova do Teorema de Tits $\quad 26$

$\begin{array}{ll}\text { Referências Bibliográficas } & 51\end{array}$ 


\section{Introdução}

Vamos dizer que a alternativa de Tits é válida para uma classe de grupos se cada grupo da classe ou é solúvel por finito (isto é, contém um subgrupo normal solúvel de índice finito) ou contém um subgrupo livre de posto no mínimo 2. Grupos livres de posto 2 contém subgrupos livres de posto enumerável. A Alternativa de Tits é assim chamada por causa do importante Teorema publicado por J. Tits [4] em 1972:

Teorema de Tits: Seja G um grupo linear finitamente gerado sobre um corpo. Então G é solúvel por finito ou G contém um subgrupo livre não cíclico.

O objetivo do presente trabalho é dar uma versão simplificada da prova de Tits. No que segue, F denotará um corpo(comutativo) arbitrário, e um grupo linear de grau n sobre $\mathrm{F}$ será um subgrupo de GL $(\mathrm{n}, \mathrm{F})$ ou, o que dá do mesmo, à um grupo de transformações lineares de um espaço vetorial n-dimensional sobre $F$. 


\section{Notações}

Apresentamos algumas notações que serão utilizadas ao longo do texto.

$\mathbb{N} \quad$ conjunto dos números naturais

$\mathbb{Z} \quad$ conjuntos dos números inteiros

$\mathbb{Q} \quad$ conjuntos dos números racionais

$\mathbb{R} \quad$ conjuntos dos números reais

$\mathbb{C} \quad$ conjuntos dos números complexos

$H \leq G \quad H$ é um subgrupo de $G$

$H \triangleleft G \quad H$ é um subgrupo normal de $G$

$G^{\prime} \quad$ subgrupo dos comutadores de $G$

$G L(n, K)$ ou $G L_{n}(K) \quad$ grupo linear geral

$M(n, F)$ ou $M_{n}(F) \quad$ conjunto das matrizes de ordem $n$ sobre o corpo $F$

$\frac{G}{H}$ ou $G / H \quad$ módulo quociente de $G$ por um subgrupo normal $H$

$(G: H)$ ou $[G: H] \quad$ índice do subgrupo $H$ em $G$

$G \times H \quad$ produto cartesiano de $G$ por $H$

$G \simeq H$ ou $G \cong H$ é isomorfo a $H$ 
$\operatorname{Aut}(G) \quad$ grupo dos automorfismos de $\mathrm{G}$

$|G| \quad$ cardinalidade do grupo $G$

$\left(x_{n}\right) \quad$ sequência com termo geral $x_{n}$

$\left(x_{n}\right) \longrightarrow y \quad$ a sequência $\left(x_{n}\right)$ converge para $y$ 


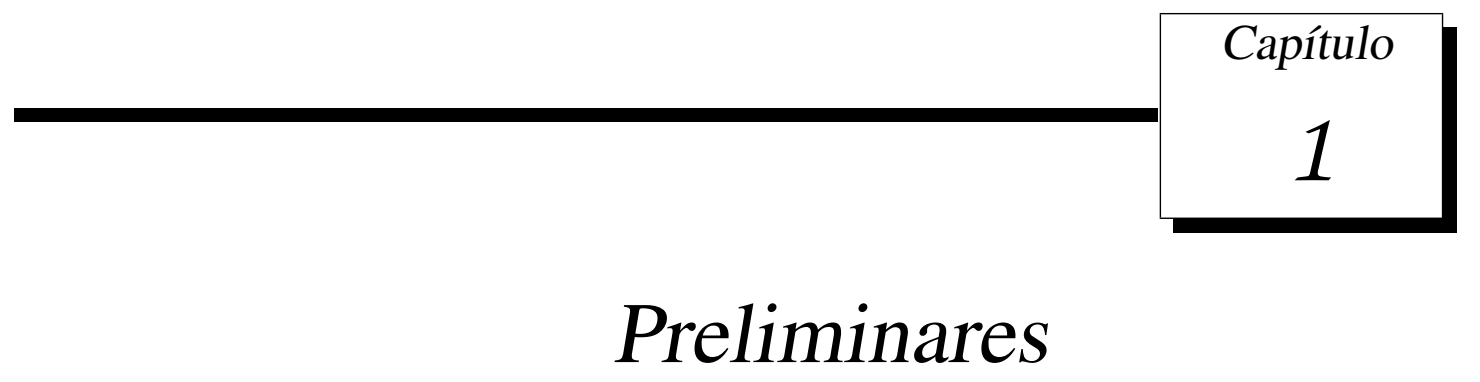

Neste capítulo introduziremos as principais definições e resultados básicos através dos quais a prova do Teorema da Alternativa de Tits será baseada. Assumiremos que o leitor tenha uma boa noção em teoria básica de grupos, anéis, módulos e corpos.

Seja $\mathrm{F}$ um corpo qualquer. Então o conjunto das matrizes quadradas de ordem $\mathrm{n}$ com coeficientes nesse corpo e de determinante diferente de zero, denotado por $G L(n, F)$, munido da operação de multiplicação usual de matrizes é um grupo, chamado de grupo linear geral. Um grupo linear será qualquer subgrupo de $G L(n, F)$.

\subsection{Topologia de Zariski}

Seja $F$ um corpo algebricamente fechado. Vamos dar agora uma breve introdução sobre um tipo especial de topologia em um grupo de matrizes arbitrário. Tal topologia é a topologia de Zariski. A topologia de Zariski é comparavelmente fraca, por exemplo, em geral ela não é Hausdorff, mas tem certas propriedades convenientes. Um subgrupo de $G L(n, F)$ o qual é fechado na topologia de Zariski é chamado de grupo algébrico. Escreveremos $F^{n}$ para denotar o espaço vetorial das $n$-uplas $x=\left(\xi_{1}, \ldots, \xi_{n}\right)$ com $\xi_{i} \in F$. Seja $X=\left(x_{1}, x_{2}, \ldots, x_{n}\right)$ uma família de $\mathrm{n}$ indeterminadas sobre $F$ e considere o anel de polinômios $F[X]=F\left[x_{1}, \ldots, x_{n}\right]$.

Definição 1.1.1 Um conjunto $A \subseteq F^{n}$ é algébrico se existe uma família $\Delta$ de polinômios em $F[X]$ tal que A é o conjunto dos zeros comuns para os polinômios em $\Delta$, isto é, $x \in A$, se e somente se, $f(x)=0, \forall f \in \Delta$.

Definição 1.1.2 Seja $B \subseteq F^{n}$. Então denotaremos por $I(B)$ o ideal de $F[X]$ dado por

$$
I(B)=\{f(X) \in F[X]: f(b)=0, \forall b \in B\}
$$


Se B for um conjunto algébrico, então B é também o conjunto algébrico correspondente a $\Delta=I(B)$.

Exemplo 1.1.1 Considere $n=2$. Então $A=\{(\xi, 0),(0, \eta): \xi, \eta \in F\}$ é um conjunto algébrico correspondente a $\Delta=\left\{x_{1}^{2} x_{2}\right\}$. Note que $I(A)=\left\langle x_{1} x_{2}\right\rangle$ não é gerado por $\Delta$.

Exemplo 1.1.2 Os conjuntos $\emptyset, F^{n} e\{x\}$ são algébricos para todo $x \in F^{n}$, pois $I(\emptyset)=F[X]$, $I\left(F^{n}\right)=(0)$ e $I(\{x\})=\left(X_{1}-\xi_{1}, \ldots, X_{n}-\xi_{n}\right)$.

Finalmente definimos a topologia de Zariski $Z$ em $F^{n}$ como segue:

Definição 1.1.3 Um subconjunto $S \subseteq F^{n}$ é um conjunto aberto na topologia de Zariski se, e somente se, $F^{n}-S$ for um conjunto algébrico. Alternativamente, os conjuntos fechados na topologia de Zariski são precisamente os conjuntos algébricos.

É fácil verificar que a topologia de Zariski é de fato uma topologia, porém tal verificação não será feita aqui. Ainda, a topologia de Zariski é $T_{1}$, já que os conjuntos unitários são fechados, pelo exemplo 1.1.2.

Definição 1.1.4 Seja $Y$ um espaço topológico qualquer. Então dado $x \in Y$, a componente conexa de $x$ é o maior conexo de $Y$ que contém $x$.

Lema 1.1.1 Seja $G$ um subgrupo de $G L(n, F)$ e seja $G_{0}$ a componente conexa da identidade em $G$. Então $G_{0}$ é um subgrupo normal de índice finito em $G$ e as componentes conexas de $G$ são as classes laterais de $G_{0}$ em $G$. Ainda mais, se $H$ for um subgrupo fechado em $G$ de indice finito em $G$, então $G_{0} \subseteq H$.

Demonstração: Ver [2], Teorema 8.5.

Exemplo 1.1.3 Seja $F=\mathbb{R}, G=\mathscr{O}_{n} \leq G L(n, \mathbb{R})$ e $H=S \mathscr{O}_{n} \leq G$, onde $\mathscr{O}_{n}=\{M \in G L(n, \mathbb{R})$ : $\operatorname{det}(M)= \pm 1\}$ e $S \mathscr{O}_{n}=\{M \in G L(n, \mathbb{R}): \operatorname{det}(M)=1\}$. Então, $S \mathscr{O}_{n}$ é a componente conexa de $\mathscr{O}_{n}$ que contém a identidade $I_{n}$. Considere a aplicação determinante det $: \mathscr{O}_{n} \longrightarrow\{ \pm 1\}$, restrita à $\mathscr{O}_{n}$. Tal aplicação é um homomorfismo sobrejetor de grupos com $\operatorname{Ker}($ det $)=S \mathscr{O}_{n}$. Logo $S \mathscr{O}_{n} \unlhd \mathscr{O}_{n} e$ pelo Teorema dos isomorfismos

$$
\frac{\mathscr{O}_{n}}{S \mathscr{O}_{n}} \simeq\{ \pm 1\}
$$

ou seja, $\left(\mathscr{O}_{n}: S \mathscr{O}_{n}\right)=2$. 
Observação 1.1.1 Se E for uma extensão de corpos de $F$, então a topologia induzida em $G \leq$ $G L(n, F)$ pela topologia de Zariski relativa à $E$ é a mesma que a induzida relativa a $F$.

Definição 1.1.5 Sejam $V$ e $W$ espaços vetoriais sobre $F$ de dimensões $n$ e $m$, respectivemente e $T$ um subconjunto de V. Uma aplicação racional de T em W é uma aplicação da forma

$$
\begin{aligned}
\phi: T \subseteq V & \longrightarrow W \\
x & \longmapsto \phi(x)=\left(r_{1}(x), \ldots, r_{m}(x)\right),
\end{aligned}
$$

onde $r_{1}, \ldots, r_{m}$ são funções racionais sobre $F$ em $n$ indeterminadas. Em outras palavras, existem polinômios $p_{i}, q_{i}$, tais que $r_{i}=\frac{p_{i}}{q_{i}}, q_{i} \neq 0$, para todo $x \in T, i=1,2, \ldots, m$

Observação 1.1.2 Toda função racional do tipo $\rho: G \leq G L(m, F) \longrightarrow G L(n, F)$ é contínua na topologia de Zariski, pois seja $A \subset F^{n}$ um conjunto fechado. Basta verificarmos que a imagem inversa $\rho^{-1}(A)$ é fechado em $G$. Com efeito, note que:

$$
\rho^{-1}(A)=\bigcap\{x \in G: g \rho(x)=0\}
$$

onde a interseção acima é sobre todos os $g\left(X_{1}, \ldots, X_{n}\right) \in I(A)$. Mas $\{x \in G: g \rho(x)=0\}$ é o conjuntos de todos os zeros comuns em $G$ dos polinômios que são os numeradores da função racional g $\rho$. Consequentemente este conjunto é fechado em S. Portanto $\rho^{-1}(A)$ é fechado em G.

Definição 1.1.6 Um Z-espaço topológico é um espaço o qual todo conjunto unitário é fechado e satisfaz a condição de cadeia descendente em conjuntos fechados, ou seja, qualquer sequência descendente $A_{1} \supseteq A_{2} \supset$... de conjuntos algébricos em $F^{n}$ é estacionária.

Definição 1.1.7 Um CZ-grupo é um Z-espaço $G$ com estrutura de grupo tal que para todo $g \in G$, as quatro aplicações dadas por

$$
x \longmapsto g x, x \longmapsto x g, x \longmapsto x^{-1} \text { e } x \longmapsto x^{-1} g x, x \in G
$$

são contínuas.

Observação 1.1.3 Sabemos que a topologia de Zariski é T1 e pelo Lema 8.2B de [2], a topologia de Zariski satisfaz a condição de cadeia descendente em conjuntos fechados. Ainda, pelo Teorema 8.4 de [2], as aplicações da definição anterior são contínuas. Logo, todo grupo linear é um CZ - grupo na topologia de Zariski. 
Lema 1.1.2 Seja H um subgrupo fechado de um CZ-grupo G. Então as seguintes afirmações são equivalentes:

1. $G^{0} \subseteq H$;

2. $(G: H)$ é finito;

3. Hé aberto.

Demonstração: Ver [5], Lema 5.3, pg. 75.

Teorema 1.1.1 Todo CZ-grupo conexo G é irredutível como espaço topológico.

Demonstração: Como por hipótese $\mathrm{G}$ é um CZ-grupo, segue que este satisfaz a condição de cadeia descendente em conjuntos fechados. Logo, $G=S_{1} \cup S_{2} \cup \cdots \cup S_{r}$ para alguma coleção finita de subconjuntos fechados irredutíveis $S_{i}$ de G. Podemos assumir claramente que $S_{i} \nsubseteq S_{j}$ para cada $i \neq j$, uma condição a qual implica pela irredutibilidade de $S_{i}$ que

$$
S_{i} \nsubseteq \bigcup_{i \neq j} S_{j}
$$

para cada i.

Suponha que $G=S \cup T$, onde $\mathrm{S}$ e T são fechados, $\mathrm{S}$ irredutível e $S \nsubseteq T$. Então $S \subseteq \cup S_{i}$ e assim $S \subseteq S_{j}$ para algum j. O conjunto irredutível $S_{j}$ também pertence à $S \cup T$ e $S_{j} \nsubseteq T$ já que $S \subseteq S_{j}$ e $S \nsubseteq T$. Consequentemente, $S_{j} \subseteq S$, o que implica $S=S_{j}$. Desse modo, a decomposição $G=\bigcup S_{i}$ é única.

Como os $S_{i}^{\prime} s$ são únicos, segue que os elementos de G permutam o conjunto $\left\{S_{1}, S_{2}, \ldots, S_{r}\right\}$ por multiplicação à direita. Suponha sem perda de generalidade que o elemento neutro $1 \mathrm{de} G$ pertença à $S_{1}$ e denote por $G_{1}$ como sendo o estabilizador de $S_{1}$ em G, ou seja, $G_{1}=\left\{g \in G: x g=x, x \in S_{1}\right\}$. Denote ainda por $\overline{G_{1}}$ como sendo o fecho de $G_{1}$ em G. Se $s \in S_{1}$, então a aplicação $x \longmapsto s x$ é um homeomorfismo de G que leva $G_{1}$ em $S_{1}$. Como $S_{1}$ é fechado, segue que $s \overline{G_{1}} \subseteq S_{1}$ para cada $s \in S_{1}$. Assim, se $g \in \overline{G_{1}}$, então $S_{1} g \subseteq S_{1}$ e como $S_{i} \nsubseteq S_{1}$ para cada $i>1$, segue que $\overline{G_{1}}=G_{1}$. Mas G é conexo por hipótese e $G_{1}$ tem índice finito em G. Logo pelo Lema 1.1.2, $G_{1}=G$. Portanto

$$
G=1 G_{1} \subseteq S_{1} G_{1}=S_{1}
$$

e assim $G=S_{1}$ o qual é irredutível. 


\subsection{Representações lineares, grupos redutíveis, irredutíveis e com- pletamente redutíveis}

Agora vamos falar um pouco sobre grupos lineares redutíveis, irredutíveis e completamente redutíveis, que são conceitos fundamentais para a discussão de tais grupos.

Definição 1.2.1 Seja $V$ um espaço vetorial de dimensão $n$ sobre um corpo $F$ qualquer e seja $G$ um subgrupo de $G L(V)$. Um subespaço $W$ de $V$ é dito um $G$-espaço, ou invariante sob $G$, se $x W \subseteq W, \forall x \in G$. Dizemos que $G$ é irredutivel se os únicos $G$-espaços forem $\{0\}$ e V. Caso contrário $G$ é redutível.

Definição 1.2.2 Seja $V$ um espaço vetorial de dimensão $n$ sobre um corpo $F$ qualquer e seja $G$ um subgrupo de $G L(V)$. Então $G$ é dito ser completamente redutivel se quando $W$ for um $G$ espaço, existe um outro $G$-espaço $U$ tal que $V=U \oplus W$. Tal subespaço $U$ é chamado de subespaço complementar de $W$.

Lema 1.2.1 Um G-subespaço de um espaço completamente redutivel V também é completamente redutível.

Demonstração: Seja W um G-subespaço de V e seja $W_{1}$ um G-subespaço de W. Então claramente $W_{1}$ também é um G-subespaço de $\mathrm{V}$ e assim existe um G-subespaço $\mathrm{W}^{\prime}$ de $\mathrm{V}$ tal que $V=W_{1} \oplus W^{\prime}$. Logo, $W_{1}^{\prime}=W^{\prime} \cap W$ é um G-subespaço de $\mathrm{W}$, e assim temos $W=W_{1} \oplus W_{1}^{\prime}$, o que prova o lema.

Definição 1.2.3 Dado um grupo abstrato $G$ e um espaço vetorial $V$, uma representação linear de G sobre Vé um homomorfismo

$$
\begin{aligned}
& \rho: G \longrightarrow G L(V) \\
& x \longmapsto \rho(x): V \longrightarrow V \\
& v \longmapsto \rho(x) v
\end{aligned}
$$

A representação $\rho$ é dita fiel se $\operatorname{Ker}(\rho)=1$. A imagem $\rho(G)$ é um grupo linear em $G L(V)$, e dizemos que $W$ é um $G$-espaço quando este for um $\rho(G)$-espaço, e chamamos $\rho$ irredutível, redutivel ou completamente redutivel se $\rho(G)$ o for. 
Teorema 1.2.1 Seja V um espaço vetorial sobre um corpo K e seja $\rho: G \longrightarrow G L(V)$ uma representação completamente redutível de G. Então V é uma soma direta de G-subespaços irredutíveis.

Demonstração: Escolha um G-subespaço $W \neq\{0\}$ de dimensão minimal entre todos os G-subespaços não nulos de V. Então W é irredutível e assim podemos encontrar um G-subespaço U de V tal que $V=W \oplus U$. Se $U=\{0\}$, então $\mathrm{V}$ é irredutível e não há nada a provar. Caso contrário, pelo lema 1.2.1, U é completamente redutível. Por outro lado, $(V: K)>(U: K)$, de onde podemos concluir por argumento indutivo que $U$ é uma soma direta de G-subespaços irredutíveis. Mas um G-subespaço de U também é um G-subespaço de V. Portanto V é uma soma direta de G-subespaços irredutíveis, como queríamos.

Definição 1.2.4 Sejam $\rho: G \longrightarrow G L(V)$ e $\sigma: G \longrightarrow G L(W)$ duas representações de $G$, onde $V$ e $W$ são espaços vetoriais sobre um mesmo corpo. Então $\rho$ é dita equivalente a $\sigma$ se existe uma aplicação linear invertível $t: V \longrightarrow W$, tal que o diagrama

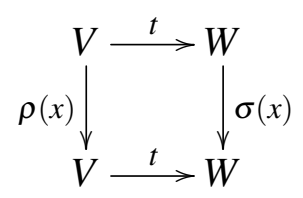

comuta para todo $x \in G$. Em particular, se $\rho$ e $\sigma$ são equivalentes, então $\operatorname{dim} V=\operatorname{dim} W . \quad A$ aplicação t será chamada de um G-homomorfismo.

Definição 1.2.5 Seja $V$ um espaço vetorial de dimensão $n$ sobre um corpo $F$ qualquer. Então $x \in G L(V)$ é unipotente se $x$ tem todos os seus n autovalores iguais a 1 , ou seja, $(x-1)^{n}=0$.

O próximo Teorema é uma consequência do Teorema de Clifford para representações completamente redutíveis ( [2], Teorema 2.2, pg.25 ).

Teorema 1.2.2 Seja G um grupo linear completamente redutivel. Então todo subgrupo subnormal de $G$ é completamente redutivel. Em particular os subgrupos normais de G são completamente redutiveis.

Demonstração: Ver Corolário 2.2, [2], pg.26. 


\subsection{Alguns grupos lineares clássicos}

Seja F um corpo. Então sabemos que $G L(n, F)$ é o grupo das unidades de $M_{n}(F)$. Segue da regra de Cramer para inversão de matrizes que uma matriz $x$ pertence à $G L_{n}(F)$, se e somente se, $\operatorname{det}(x) \neq 0$.

O conjunto de todas as matrizes triangulares (inferiores e superiores ) de $G L(n, F)$ formam um subgrupo $T L(n, F)$ chamado de grupo triangular. As matrizes em $T L(n, F)$ com todas as entradas da diagonal principal iguais à $1 \in F$, formam o grupo triangular especial $S T L(n, F)$. O conjunto de todas as matrizes diagonais de $G L(n, F)$ também formam um grupo chamado de grupo diagonal e denotado por $\operatorname{Diag}(n, F)$.

Temos o seguinte Lema que será útil no capítulo 3:

Lema 1.3.1 Seja $F$ um corpo e $F^{*}$ seu grupo multiplicativo. Então:

1. $\operatorname{Diag}(n, F)=F^{*} \times \cdots \times F^{*}(n$ vezes $)$.

2. O subgrupo derivado de $T L(n, F)$ é o $\operatorname{STL}(n, F)$.

3. $\operatorname{STL}(n, F)$ é nilpotente de classe $n-1$ e solúvel de comprimento l, onde $2^{l-1}<n \leq 2^{l}$.

4. $T L(n, F)$ é o normalizador de $\operatorname{STL}(n, F)$ em $G L(n, F)$.

Demonstração: Ver Lema 1.3 de [2], pg. 12.

\subsection{Lema do Ping-Pong}

Vamos definir o que são grupos livres e produto livre de dois grupos, dando a idéia da construção de ambos e por fim, enunciar e demonstrar o lema do ping-pong.

Definição 1.4.1 Um grupo $G$ é dito livre se existe um subconjunto $S$ de G tal que qualquer elemento de $G$ pode ser escrito, de uma única maneira, como um produto finito de elementos de $S$ e seus inversos.

Exemplo 1.4.1 O grupo aditivo $(\mathbb{Z},+)$ é livre, tomando $S=\{1\}$.

Teorema 1.4.1 Dado um grupo G e um conjunto gerador livre $S$, sempre existe o grupo livre $G_{S}$. 
Demonstração: A construção pode ser feita da seguinta maneira: S é um conjunto de símbolos e vamos supor que para todo $s \in S$, existe o seu correspondente "inverso" simbólico " $s$ "1", em um conjunto $S^{-1}$. Seja $T=S \bigcup S^{-1}$ e defina uma palavra em $\mathrm{S}$ como sendo qualquer produto de elementos de T. A palavra vazia é a palavra que não tem símbolos. Por exemplo, se $S=\{a, b, c\}$, então $T=\left\{a, a^{-1}, b, b^{-1}, c, c^{-1}\right\}$ e $a b c^{-1} c a^{-1} c$ é uma palavra em S. Se tivermos um par $s s^{-1}$, podemos omití-lo da palavra. Logo, $a b c^{-1} c a^{-1} c=a b a^{-1} c$ é a palavra simplificada. Uma palavra que não pode ser simplificada é chamada reduzida. O grupo livre $G_{S}$ é definido como sendo o grupo de todas as palavras reduzidas em $S$, com a operação de concatenação de palavras, seguida de redução, se necessário. A identidade é a palavra vazia.

Definição 1.4.2 Seja $S$ um conjunto não vazio de subgrupos de um grupo G. Então G é o produto livre $\prod^{*}\{H: H \in S\}$ do conjunto $S$ de subgrupos se, e somente se, cada $g \in G, g \neq 1$, puder ser escrito unicamente da forma:

$$
g=x_{1} \ldots x_{k}, \quad e \neq x_{i} \in H_{i} \in S, \quad H_{i} \neq H_{i+1} .
$$

Usaremos a notação $G=H_{1} * H_{2} * \ldots$

Teorema 1.4.2 Dados dois grupos $G$ e $H$, sempre existe o produto livre $G * H$.

Demonstração: A construção pode ser feita da seguinte forma: sejam $\mathrm{G}$ e $\mathrm{H}$ os grupos em questão. Então uma palavra em $\mathrm{G}$ e $\mathrm{H}$ é um produto da forma $s_{1} s_{2} \ldots s_{n}$, onde cada $s_{i}$ pertence à $\mathrm{G}$ ou a $\mathrm{H}$. Uma palavra pode ser reduzida removendo os elementos identidade de $\mathrm{G}$ e $\mathrm{H}$. Toda palavra reduzida é um produto alternado de elementos de G e elementos de $\mathrm{H}$, como por exemplo $g_{1} h_{1} g_{2} h_{2} \ldots g_{k} h_{k}$. O produto livre $G * H$ é o grupo cujos elementos são as palavras reduzidas em $\mathrm{G}$ e $\mathrm{H}$, sobre a operação de concatenação de palavras seguida de redução, se necessário.

Exemplo 1.4.2 Se G for o grupo cíclico infinito $\langle x\rangle$ e H for o grupo cíclico infinito $\langle y\rangle$, então todo elemento de $G * H$ é um produto alternado de potências de x com potências de y. Neste caso, $G * H$ é isomorfo ao grupo livre gerado por $x$ e $y$.

Lema 1.4.1 (Lema do Ping-Pong) Seja $G$ um grupo agindo em um conjunto $\Omega$ e sejam A e B dois subgrupos de G. Suponha que existam conjuntos não vazios $\Delta, \Gamma \subseteq \Omega$ tais que:

1. $\Delta \bigcap \Gamma=\emptyset$;

2. $\Gamma a \subseteq \Delta e \Delta b \subseteq \Gamma$, para todo $a \in A, b \in B$ não triviais; 
3. $\Delta a \bigcap \Delta \neq \emptyset$, para todo $a \in A$.

Então o subgrupo $\langle A, B\rangle$ é o produto livre $A * B$.

Demonstração: É suficiente mostrarmos que $1 \in G$ não pode ser escrito como $1=a_{1} b_{1} a_{2} b_{2} \ldots a_{k} b_{k}$, $\operatorname{com} k \geq 1$ e $a_{i} \in A, b_{i} \in B$ não triviais. De fato, suponha isto verdade. Então sejam $a_{1} b_{1} a_{2} b_{2} \ldots a_{k} b_{k}$, $\bar{a}_{1} \bar{b}_{1} \bar{a}_{2} \bar{b}_{2} \ldots \bar{a}_{r} \bar{b}_{r} \in\langle A, B\rangle, k, r \geq 1 \mathrm{com}$

$$
a_{1} b_{1} a_{2} b_{2} \ldots a_{k} b_{k}=\bar{a}_{1} \bar{b}_{1} \bar{a}_{2} \bar{b}_{2} \ldots \bar{a}_{r} \bar{b}_{r}
$$

Assim, $1=b_{k}^{-1} a_{k}^{-1} \ldots b_{1}^{-1} a_{1}^{-1} \bar{a}_{1} \bar{b}_{1} \ldots \bar{a}_{r} \bar{b}_{r}$, o que implica $r=k, a_{i}=\bar{a}_{i}$ e $b_{i}=\bar{b}_{i}$. Logo $\langle A, B\rangle=$ $A * B$. Mostremos então a asserção que falta. Seja $g=a_{1} b_{1} a_{2} b_{2} \ldots a_{k} b_{k}, k \geq 1, a_{i} \in A, b_{i} \in B$ não triviais. Queremos mostrar que $g \neq 1$. Com efeito, como $a_{1} \in A$, segue por 3 que existe $\delta \in \Delta$ tal que $\delta a_{1} \in \Delta$. Logo $\delta g \in \Gamma$, pois note que:

$$
\delta g=\delta a_{1} b_{1} a_{2} b_{2} \ldots a_{k} b_{k}=\underbrace{\left(\delta a_{1}\right)}_{\in \Delta} b_{1} a_{2} b_{2} \ldots a_{k} b_{k}
$$

Mas $\delta a_{1} \in \Delta$, o que implica por 2 que $\left(\delta a_{1}\right) b_{1} \in \Gamma$, o que implica novamente por 2 que $\underbrace{\left(\delta a_{1} b_{1}\right)}_{\in \Gamma} a_{2} \in$ $\Delta$. Continuando este processo, obtemos:

$$
\delta a_{1} b_{1} a_{2} b_{2} \ldots a_{k} \in \Delta
$$

o que implica por 2 que $\delta a_{1} b_{1} a_{2} b_{2} \ldots a_{k} b_{k}=\delta g \in \Gamma$. Daí, $\delta \in \Delta$ e $\delta g \in \Gamma$. Logo, se $g=1$ então $\delta \in \Delta \cap \Gamma$, o que por 3 nos dá um absurdo. Portanto $g \neq 1$, como queríamos.

Exemplo 1.4.3 Tome $G=G L(2, \mathbb{C})$ agindo sobre $\mathbb{C}^{2}$ e definimos

$$
a=\left[\begin{array}{ll}
1 & 0 \\
\theta & 1
\end{array}\right] \text { e } b=\left[\begin{array}{ll}
1 & \theta \\
0 & 1
\end{array}\right], \theta \in \mathbb{C} .
$$

Tomando $A=\langle a\rangle, B=\langle b\rangle, \Gamma=\left\{(\alpha, \beta) \in \mathbb{C}^{2}:|\alpha|<|\beta|\right\}$ e $\Delta=\left\{(\alpha, \beta) \in \mathbb{C}^{2}:|\alpha|>|\beta|\right\}$ e supondo $|\theta|>2$, é fácil verificar que $G, H, \Delta e \Gamma$ satisfazem as três condições do Lema do Ping-Pong. Logo $\langle A, B\rangle=\langle a, b\rangle$ é um grupo livre de posto 2 . 


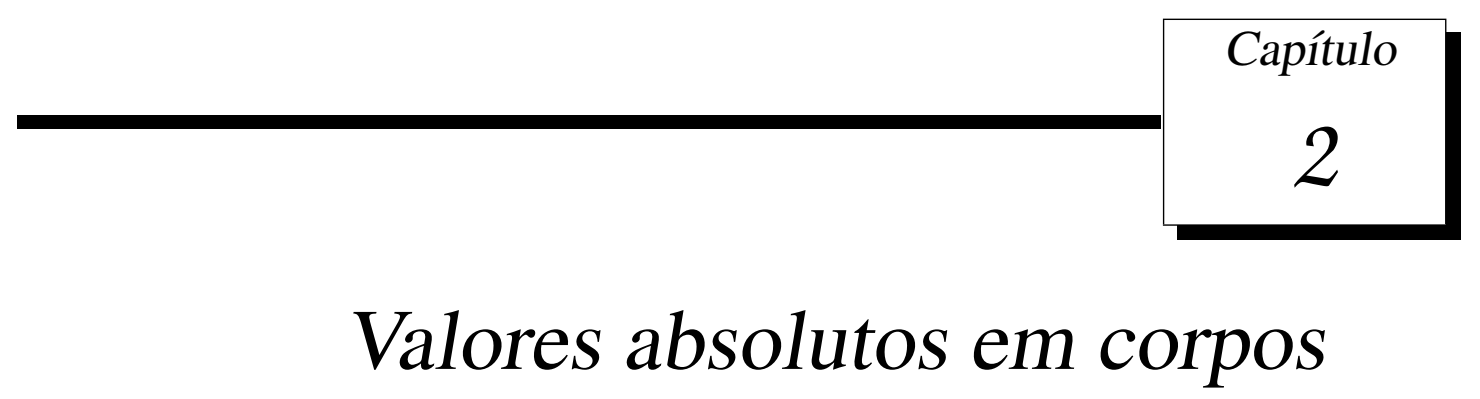

\subsection{Valores absolutos em um corpo}

Seja $\mathrm{K}$ um corpo e seja $\mathbb{R}_{+}=\{x \in \mathbb{R}: x \geq 0\}$ o conjunto dos números reais não negativos. Vamos começar definindo um valor absoluto em $\mathrm{K}$ e explorar as possibilidades implícitas na definição.

Definição 2.1.1 Um valor absoluto em $K$ é uma função

$$
||: K \longrightarrow \mathbb{R}_{+}
$$

que satisfaz as seguintes condições:

1. $|x| \geq 0 e|x|=0 \Longleftrightarrow x=0, \forall x \in K$;

2. $|x y|=|x||y|, \forall x, y \in K$

3. $|x+y| \leq|x|+|y|, \forall x, y \in K$.

Diremos que um valor absoluto em K é não-arquimediano se satisfazer a condição adicional:

4. $|x+y| \leq \max \{|x|,|y|\}, \forall x, y \in K$.

Caso contrário, diremos que o valor absoluto é arquimediano. Note que a condição 4 implica 3 , pois $\max \{|x|,|y|\}$ é certamente menor do que a soma $|x|+|y|$. 
Exemplo 2.1.1 O exemplo mais óbvio é considerar $K=\mathbb{Q}$ e tomar o valor absoluto usual || definido por:

$$
|x|= \begin{cases}x, & \text { se } x \geq 0 \\ -x, & \text { se } x<0\end{cases}
$$

É fácil ver que este valor absoluto é arquimediano.

Exemplo 2.1.2 Outro exemplo, num corpo qualquer, é o chamado valor absoluto trivial, definido por:

$$
|x|= \begin{cases}1, & \text { se } x \neq 0 \\ 0, & \text { se } x=0\end{cases}
$$

Definição 2.1.2 Seja $K$ um corpo. Uma aplicação $v: K \longrightarrow \mathbb{Z} \cup\{\infty\}$ é uma valorização sobre $K$ se, $\forall a, b \in K$ :

1. $v(a)=\infty \Leftrightarrow a=0$;

2. $v(a b)=v(a)+v(b)$;

3. $v(a+b) \geq \min \{v(a), v(b)\}$.

Vamos introduzir agora um exemplo de valorização, a qual será utilizada futuramente.

Exemplo 2.1.3 Tome $K=\mathbb{Q}$ e escolha um primo $p \in \mathbb{Z}$. Seja $n \in \mathbb{Z}$. Então $n$ pode ser escrito unicamente na forma $n=p^{v} . n^{\prime}$, com $p \nmid n^{\prime}$. Como $v$ é determinado por $p$ e $n$, faz sentido definirmos uma função $v_{p}$ por $v_{p}(n)=v$, tal que $v_{p}(n)$ é a multiplicidade de p como divisor de $n$. Em outras palavras, escolhido o primo $p \in \mathbb{Z}$, a valorização $p$-ádica em $\mathbb{Z}$ é a função

$$
\begin{aligned}
v_{p}: \mathbb{Z}-\{0\} & \longrightarrow \mathbb{R} \\
n & \longmapsto v_{p}(n)
\end{aligned}
$$

onde $v_{p}(n)$ é o único inteiro positivo que satisfaz $n=p^{v_{P}(n)} \cdot n^{\prime}$, com $p \nmid n^{\prime}$. Podemos estender $v_{p}$ para o corpo dos racionais $\mathbb{Q}$ da seguinte maneira: se $x=\frac{a}{b} \in \mathbb{Q}-\{0\}$, então $v_{p}(x)=v_{p}(a)-$ $v_{p}(b)$.

Definição 2.1.3 Para todo $x \in \mathbb{Q}$, definimos o valor absoluto p-ádico de x por:

$$
|x|_{p}= \begin{cases}p^{-v_{p}(x)}, & x \neq 0 \\ 0, & x=0\end{cases}
$$


Proposição 2.1.1 A função ||$_{p}$ é um valor absoluto não-arquimediano.

Demonstração: Ver [6], Proposição 2.1.5.

\subsection{Topologia induzida por um valor absoluto}

A grande vantagem de um valor absoluto é que este nos dá uma noção de "tamanho". Em outras palavras, uma vez que temos um valor absoluto, podemos usá-lo para medir distâncias entre números, isto é, colocar uma métrica no nosso corpo em questão. Tendo uma métrica, podemos definir abertos e fechados e em geral investigar a topologia do nosso corpo.

Definição 2.2.1 Seja K um corpo e || um valor absoluto em K. Definimos a distância d $(x, y)$ entre dois elementos $x$ e y de K por

$$
d(x, y)=|x-y|
$$

A função $d(x, y)$ é chamada métrica induzida pelo valor absoluto. Ainda, $d(x, y)$ satisfaz, $\forall x, y, z \in$ $K$ :

1. $d(x, y) \geq 0$ e $d(x, y)=0 \Leftrightarrow x=y$;

2. $d(x, y)=d(y, x)$;

3. $d(x, z) \leq d(x, y)+d(y, z)$.

Logo, $d(x, y)$ é uma métrica em K. Portanto, podemos dizer que um corpo com um valor absoluto pode ser visto como um espaço métrico definindo $d(x, y)=|x-y|$ como sendo sua métrica.

Definição 2.2.2 Seja $K$ um corpo com um valor absoluto || . Seja $a \in K$ e $r \in \mathbb{R}_{+}$. A bola aberta de raio r e centro a é o conjunto

$$
B(a, r)=\{x \in K: d(x, a)<r\}=\{x \in K:|x-a|<r\} .
$$

A bola fechada de raio r e centro a é o conjunto

$$
\bar{B}(a, r)=\{x \in K: d(x, a) \leq r\}=\{x \in K:|x-a| \leq r\} .
$$


Estas são as definições usuais em um espaço métrico. As bolas abertas são os protótipos de conjuntos abertos e as bolas fechadas de conjuntos fechados. Ainda, sendo $x \in K$, então uma base de vizinhanças de $x$ são as bolas abertas de centro $x$ e raio $r, r>0$. Sendo assim, um corpo K com a topologia induzida por um valor absoluto é um corpo topológico, isto é, as operações de soma e produto são contínuas. Para mais detalhes, ver [1], Capítulo 2 - $\$ 4$.

Definição 2.2.3 Dois valores absolutos $\|_{1}$ e $\left.\right|_{2}$ em um corpo $K$ são ditos equivalentes se eles definem a mesma topologia em $K$, isto é, se todo conjunto que for aberto com respeito a $\|_{1}$ também é aberto com respeito a $\|_{2}$ e vice-versa.

Um critério mais acessível é dado pelo seguinte resultado:

Proposição 2.2.1 Sejam $\left\|_{1} e\right\|_{2}$ dois valores absolutos em um corpo K. Então as seguintes afirmações são equivalentes:

1. ||$_{1}$ e $\|_{2}$ são valores absolutos equivalentes;

2. $\forall x \in K$ temos $|x|_{1}<1 \Leftrightarrow|x|_{2}<1$;

3. existe um número real positivo $\alpha$ tal que $\forall x \in K,|x|_{1}=|x|_{2}^{\alpha}$.

Demonstração: Ver [6], Lema 3.2.1.

\subsection{Completamento: os corpos p-ádicos $\mathbb{Q}_{p}$}

Sabemos que todo corpo $\mathrm{K}$ com um valor absoluto || pode ser visto como um espaço métrico cuja métrica é a induzida pelo valor absoluto ||. Faz sentido então questionar se um determinado corpo com um valor absoluto é completo. Para isto, temos as seguintes definições:

Definição 2.3.1 Seja K um corpo e seja || um valor absoluto em K. Então:

- Uma sequência de elementos $x_{n} \in K$ é chamada uma sequência de Cauchy se $\forall \varepsilon>0$, pudermos encontrar $M>0$ suficientemente grande tal que $\left|x_{n}-x_{m}\right|<\varepsilon$ quando $m, n \geq M$.

- O corpo K é dito completo com respeito a || se toda sequência de Cauchy de elementos de $K$ for convergente. 
- Um subconjunto $S \subset K$ é dito denso em $K$ se toda bola aberta em $K$ contém ao menos um elemento de $S$. Em símbolos, $\forall x \in K e \forall \varepsilon>0 \Longrightarrow B(x, \varepsilon) \cap S \neq \emptyset$.

Teorema 2.3.1 O corpo dos números racionais $\mathbb{Q}$ não é completo com respeito a nenhum de seus valores absolutos não-triviais.

Demonstração: Ver [6], Lema 3.2.3.

Como $\mathbb{Q}$ não é completo, nosso objetivo agora será construir um completamento para $\mathbb{Q}$, ou seja, queremos “acrescentar à $\mathbb{Q}$ os limites de todas as sequências de Cauchy de elementos de $\mathbb{Q}$ ”.

Definição 2.3.2 Seja ||$=||_{p}$ um valor absoluto não-arquimediano em $\mathbb{Q}$. Vamos denotar por $\mathscr{C}$, ou $\mathscr{C}_{p}(\mathbb{Q})$ se quisermos enfatizar p e $\mathbb{Q}$, como sendo o conjunto de todas as sequências de Cauchy de elementos de $\mathbb{Q}$ :

$$
\mathscr{C}=\mathscr{C}_{p}(\mathbb{Q})=\left\{\left(x_{n}\right):\left(x_{n}\right) \text { é uma sequência de Cauchy com respeito a }||_{p}\right\} .
$$

Definindo as operações de soma $(+)$ e produto $(\cdot)$ de sequências por:

$$
\begin{gathered}
\left(x_{n}\right)+\left(y_{n}\right)=\left(x_{n}+y_{n}\right) \\
\left(x_{n}\right) \cdot\left(y_{n}\right)=\left(x_{n} y_{n}\right),
\end{gathered}
$$

isto nos dá uma estrutura para $\mathscr{C}$ de anel comutativo com unidade. Ainda, $\mathscr{C}$ não é um corpo pois contém divisores de zero. Para mais detalhes, ver [6], pg.53.

Note agora que $\mathscr{C}$ contém $\mathbb{Q}$, pois se $x \in \mathbb{Q}$, então a sequência $x, x, x, x, \ldots$ é certamente de Cauchy, a qual chamaremos de sequência constante associada a $x$ e denotada por $(x)$. Assim, o mapa $x \longmapsto(x)$ é uma inclusão de $\mathbb{Q}$ em $\mathscr{C}$.

No entanto, o principal problema com $\mathscr{C}$ é que este ainda não nos dá uma idéia boa para "adicionar à $\mathbb{Q}$ todas as sequências de Cauchy", pois diferentes sequências de Cauchy cujos termos são próximos uns aos outros "deveriam" ter o mesmo limite, mas estas são objetos diferentes em $\mathscr{C}$. Este tipo de situação nos induz a identificar duas sequências as quais "deveriam" ter o mesmo limite, o que significa que devemos quocientar $\mathscr{C}$ pelo seguinte ideal $\mathscr{N} \subset \mathscr{C}$, onde

$$
\mathscr{N}=\left\{\left(x_{n}\right): x_{n} \longrightarrow 0\right\}=\left\{\left(x_{n}\right): \lim _{n \rightarrow \infty}\left|x_{n}\right|_{p}=0\right\}
$$

Temos que $\mathscr{N}$ é ideal maximal de $\mathscr{C}$. Para isto, ver [6], Lema 3.2.8. Assim, segue a definição de $\mathbb{Q}_{p}$, para algum p primo. 
Definição 2.3.3 Definimos o corpo dos números p-ádicos como sendo o quociente do anel $\mathscr{C}$ pelo ideal maximal $\mathscr{N}$ :

$$
\mathbb{Q}_{p}=\frac{\mathscr{C}}{\mathscr{N}} .
$$

Note que duas sequências constantes distintas nunca diferem por um elemento de $\mathscr{N}$. Estas diferem-se por outra sequência constante. Consequentemente, temos a inclusão $i: \mathbb{Q} \longrightarrow \mathbb{Q}_{p}$, levando $x \in \mathbb{Q}$ na classe de equivalência da sequência constante $(x)$.

Seja agora $\lambda \in \mathbb{Q}_{p}$ e $\left(x_{n}\right)$ qualquer sequência de Cauchy representando a classe de equivalência $\lambda$. Definindo

$$
|\lambda|_{p}=\lim _{n \mapsto \infty}\left|x_{n}\right|_{p}
$$

temosque ||$_{p}$ é um valor absoluto não-arquimediano em $\mathbb{Q}_{p}$, o qual estende o valor absoluto p-ádico em $\mathbb{Q}$. Ainda, $\mathbb{Q}_{p}$ é completo com respeito a este valor absoluto. Para mais detalhes, ver [6], pg $56-58$.

Um resultado mais geral é o seguinte:

Teorema 2.3.2 Todo corpo K com um valor absoluto tem um completamento $\widehat{K}$, que também é um corpo tal que $\widehat{K} \supset K$.

Demonstração: Ver [7], Teorema 9.7.

\subsection{Extensões finitas de corpos completos}

Um dos problemas centrais em teoria de valorização é: Dado um corpo K com um valor absoluto || e uma extensão finita de corpos $K \subseteq E$, podemos estender || à um valor absoluto em E? Vamos considerar este problema para o caso do nosso corpo $\mathrm{K}$ ser completo com respeito ao seu respectivo valor absoluto. Primeiro vamos falar sobre extensões de $\mathbb{Q}_{p}$ (o qual já sabemos que é completo com respeito ao valor absoluto ||$_{p}$ ) e depois de um modo mais geral, veremos dois resultados que garantem a existência da extensão dessas normas(valores absolutos) para K completo, sendo tais valores absolutos arquimedianos ou não-arquimedianos.

Seja então E um corpo contendo $\mathbb{Q}_{p}$. Isto significa que E é um espaço vetorial sobre $\mathbb{Q}_{p}$, e dizemos que E é uma extensão finita de $\mathbb{Q}_{p}$ se sua dimensão como um $\mathbb{Q}_{p}$-espaço vetorial for finita. Denotaremos por $\left[E: \mathbb{Q}_{p}\right]=\operatorname{dim}_{\mathbb{Q}_{p}} E$ e chamaremos este número de grau de E sobre $\mathbb{Q}_{p}$.

Queremos valores absolutos em E que estendem o valor absoluto p-ádico em $\mathbb{Q}_{p}$, ou seja, estamos procurando por uma função

$\|: K \longrightarrow \mathbb{R}_{+}$a qual é um valor absoluto, 
e que também satisfaça a condição $|\lambda|=|\lambda|_{p}$, quando $\lambda \in \mathbb{Q}_{p}$.

Até aqui, há várias coisas para se notar. Primeiro que qualquer função do tipo anterior será uma norma em E como um $\mathbb{Q}_{p}$-espaço vetorial. Segundo, o valor absoluto || terá que ser nãoarquimediano, pois estes dependem somente dos valores absolutos dos elementos de $\mathbb{Z}$, os quais estão em $\mathbb{Q}_{p}$.

A proposição a seguir mostra que se tal valor absoluto existir, então este deve ter certas propriedades. Depois, usaremos tais propriedades para obter uma construção à qual mostra que a extensão que estamos procurando existe de fato.

Proposição 2.4.1 Seja E uma extensão finita de $\mathbb{Q}_{p}$. Se existir um valor absoluto || em E estendendo o valor absoluto p-ádico em $\mathbb{Q}_{p}$, então:

1. E é completo com respeito à || ;

2. Podemos considerar o limite de uma sequência em E tomando os limites dos coeficientes com respeito a qualquer base dada $\left\{x_{1}, x_{2}, \ldots, x_{n}\right\}$ de E como um $\mathbb{Q}_{p}$-espaço vetorial. Em particular, a topologia em E induzida por || é simplesmente a única topologia em E como um $\mathbb{Q}_{p}$-espaço vetorial normado, e portanto é independente da escolha do valor absoluto.

Demonstração: Ver [6], Proposição 5.3.1.

Corolário 2.4.1 Existe no máximo um valor absoluto em E estendendo o valor absoluto p-ádico em $\mathbb{Q}_{p}$.

Demonstração: Ver [6], Corolário 5.3.2. 
Sabemos até agora que pode existir no máximo uma extensão do valor absoluto p-ádico para E e que se este existir, então E será completo com respeito a esta extensão. Por outro lado, nenhuma dessas conclusões garantem que tal extensão existe. Para mostrar a existência de tal valor absoluto precisamos fazer uma construção. Antes, uma consequência importante da unicidade que deve ser notada é que: Suponha que tenhamos duas extensões E e L de $\mathbb{Q}_{p}$, uma contendo a outra, de modo que $\mathbb{Q}_{p} \subset L \subset E$, e suponha que tenhamos achado valores absolutos $\left.\right|_{L}$ em L e ||$_{E}$ em E, ambos estendendo o valor absoluto p-ádico em $\mathbb{Q}_{p}$. A restrição de $\left.\right|_{E}$ aos elementos de L é um valor absoluto em $\mathrm{L}$, o qual também estende o valor absoluto p-ádico em $\mathbb{Q}_{p}$. Logo, pela unicidade, este deve ser o mesmo que ||$_{L}$. Em outras palavras, se $x \in L \subset E$, então $|x|_{L}=|x|_{E}$.

Vamos então à construção do valor absoluto em E. Sejam K e E corpos, $\operatorname{com} K \subset E$ e $[E: K]$ finito. Vamos assumir que nossos corpos têm característica 0 . Seja $C$ um corpo algebricamente fechado contendo $\mathrm{K}$ ( ou fixe uma inclusão $\mathrm{K} \hookrightarrow C$ e identifique $\mathrm{K}$ com sua imagem ). Diremos que a extensão de corpos $K \subset E$ é normal se todos os ( necessariamente injetivos ) homomorfismos $\sigma: E \longrightarrow C$ os quais induzem a identidade em $\mathrm{K}$ tiverem a mesma imagem. Uma outra maneira de dizer isto é identificar $\mathrm{E}$ com uma de suas imagens, e depois dizer que $K \subset E$ é normal se todo $\sigma$ mapeia E em E.

Em um simples diagrama, $\sigma$ se encaixa da seguinte maneira:

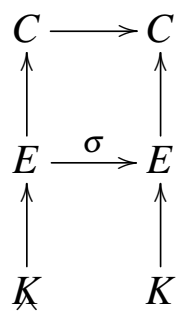

onde as setas verticais são inclusões.

Quando $K \subset E$ for normal, é claro que a escolha de $\mathrm{C}$ não importa, já que todo $\sigma$ mapeia $\mathrm{E}$ em $\mathrm{E}$. Chamamos um mapa $\sigma: E \longrightarrow E$ o qual induz a identidade em $\mathrm{K}$ por automorfismo da extensão $K \subset E$. Sabe-se que quando $K \subset E$ é normal e de característica 0 , os automorfismos de $K \subset E$ formam um grupo finito cuja ordem é igual ao grau $[E: K]$. No caso em que a característica dos corpos forem primos, precisamos da condição extra da extensão $K \subset E$ ser separável. Tal grupo é chamado o grupo de Galois da extensão $K \subset E$. Para mais detalhes, ver [3], capítulos V e VI.

Sabe-se também que dada qualquer extensão finita $K \subset E$, existe uma extensão normal finita de $\mathrm{K}$ contendo E. A menor delas é chamada fecho normal de $K \subset E$. Isto será útil no que segue, pois vamos poder assumir que E é extensão normal de $\mathrm{K}$ a fim de construir o valor absoluto de um elemento $x \in E$. O fato crucial que precisaremos é que existe uma função

$$
N_{E / K}: E \longrightarrow K
$$


a qual é chamada de norma de E para K, e é muito útil pois nos permite de um modo natural "descer" dos elementos do corpo maior E para os elementos de K. Tal função norma pode ser definida de várias maneiras, cada qual útil em determinados contextos; aqui estão três:

- Tome $\alpha \in E$. Veja E como um K-espaço vetorial de dimensão finita, e considere o mapa K-linear

$$
\begin{aligned}
T_{\alpha}: E & \longrightarrow E \\
x & \longmapsto \alpha x
\end{aligned}
$$

Como $T_{\alpha}$ é K-linear, $\forall \alpha \in E$, segue que $T_{\alpha}$ corresponde à uma matriz. Então defina $N_{E / K}(\alpha)$ como sendo o determinante desta matriz.

- Tome $\alpha \in E$ e considere a subextensão $K(\alpha)$, isto é, o menor corpo contendo $\mathrm{K}$ e $\alpha$, que é claramente um subcorpo de E. Seja $r=[E: K(\alpha)]$ e seja

$$
f(X)=X^{n}+a_{n-1} X^{n-1}+\cdots+a_{1} X+a_{0} \in K[X]
$$

o polinômio minimal de $\alpha$ sobre K. Defina $N_{E / K}(\alpha)=(-1)^{n r} a_{0}^{r}$.

- Suponha que a extensão $K \subset E$ seja normal. Então defina $N_{E / K}(\alpha)$ como sendo o produto de todos os $\sigma(\alpha)$, onde $\sigma$ percorre o conjunto (finito) de todos os automorfismos de $K \subset E$.

Estas três definições são equivalentes, ou seja, no final dão a mesma resposta. Para mais detalhes ver [6], pg.147-148.

Com isso, temos o seguinte resultado, o qual nos dá uma fórmula explícita para o cálculo da única norma (valor absoluto) em um corpo $K \supset \mathbb{Q}_{p}$ que estende o valor absoluto p-ádico de $\mathbb{Q}_{p}$, sendo a extensão $\mathbb{Q}_{p} \subset K$ não necessariamente normal, bastando considerarmos, neste caso, o fecho normal de $\mathbb{Q}_{p} \subset K$.

Teorema 2.4.1 Seja $\mathbb{Q}_{p} \subset K$ uma extensão finita de $\mathbb{Q}_{p}$ de grau $n$. Então a função ||$: K \longrightarrow \mathbb{R}_{+}$ definida por

$$
|x|=\sqrt[n]{\left|N_{K / \mathbb{Q}_{p}}(x)\right|_{p}}
$$

é o único valor absoluto em $K$ que estende o valor absoluto $p$-ádico de $\mathbb{Q}_{p}$. Ainda, || é nãoarquimediano.

Demonstração: Ver [6], Lema 5.3.3, Proposição 5.3.4 e Teorema 5.3.5. agora sabemos explicitamente a única maneira de se estender o valor absoluto p-ádico de $\mathbb{Q}_{p}$ em 
um corpo $\mathrm{K}$ contendo $\mathbb{Q}_{p}$. Observe que até aqui, foi considerado o corpo completo menor como sendo $\mathbb{Q}_{p}$. Em geral, para qualquer corpo completo $\mathrm{K}$, temos os seguintes resultados:

Teorema 2.4.2 Seja || um valor absoluto não trivial arquimediano em $K$, tal que $K$ é completo com respeito à ||, e seja E uma extensão de corpos finita de K. Então || pode ser estendido a um valor absoluto em E dado unicamente por:

$$
|x|=\left|N_{E / K}(x)\right|^{\frac{1}{[E: K]}} .
$$

Ainda, E é completo com respeito à este valor absoluto.

Demonstração: Ver [7], Teorema 9.9.

Teorema 2.4.3 Seja $K$ um corpo com um valor absoluto não-arquimediano ||, $K$ completo com respeito à || e seja E uma extensão finita de F. Então || pode ser estendido a um valor absoluto (necessariamente não-arquimediano) em E, dado unicamente por:

$$
|x|=\left|N_{E / K}(x)\right|^{\frac{1}{[E: K]}} .
$$

Ainda, E é completo com respeito a este valor absoluto.

Demonstração: Ver [7], Teorema 9.12.

Observação 2.4.1 De um modo ainda mais geral, podemos considerar $K$ um corpo qualquer com um valor absoluto ||, ou seja, não necessariamente $K$ sendo completo com respeito ao seu valor absoluto, de tal maneira que se tivermos uma extensão finita $K \subset E$, então é possível estendermos | | de K para E. Para mais detalhes, ver [7], pg.565 e Teorema 9.13, pg.566, ou capítulo XII - seção 3 de [3].

Definição 2.4.1 Seja $B \supset$ A uma extensão de anéis. Um elemento $b \in B$ é dito integral sobre A se ele for raiz de um polinômio mônico em $A[x]$, isto é, existe $n \geq 1$ tal que

$$
b^{n}+a_{n-1} b^{n-1}+a_{n-2} b^{n-2}+\cdots+a_{0}=0 \quad\left(a_{i} \in A\right) .
$$

Um número complexo $\theta$ que for integral sobre $\mathbb{Z}$ é chamado de inteiro algébrico.

Vejamos agora três resultados que serão úteis futuramente: 
Teorema 2.4.4 Todo corpo K com uma valor absoluto || pode ser imerso em um corpo completo $\bar{K}$ com valor absoluto || estendendo o valor absoluto de $K$ de tal modo que $\bar{K}$ é o fecho de $K$ com respeito a ||. Ainda, $\bar{K}$ é único a menos de isomorfismo.

Demonstração: Ver [1], pg. 47.

Teorema 2.4.5 (Kronecker) Seja $K \supset \mathbb{Q}$ uma extensão finita de corpos. Seja ainda $\xi$ um inteiro algébrico tal que $|\sigma(\xi)|=1$ para toda imersão $\sigma: K \hookrightarrow \mathbb{C}$. Então $\xi$ é uma raiz da unidade.

Demonstração: Ver [8], Proposição 6.75, pg. 267.

Teorema 2.4.6 Seja $K$ um corpo, E uma extensão algébrica de $K$ e $\sigma: K \rightarrow L$ uma imersão de $K$ em um corpo algebricamente fechado L. Então existe uma extensão de $\sigma$ para uma imersão de $E$ em L. Se E é algebricamente fechado e L é algébrico sobre $\sigma(K)$, então qualquer extensão de $\sigma$ é um isomorfismo de E para $L$.

Demonstração: Ver [3], Teorema 2.8, pg.233.

\subsection{Corpos locais}

Seja K um corpo com valor absoluto não-trivial não-arquimediano || . Tome $\mathscr{O}=\{x \in$ $K:|x| \leq 1\}$ e $\mathfrak{p}=\{x \in K:|x|<1\}$. Segue das propriedades de valor absoluto não-arquimediano que $\mathscr{O}$ é um subanel de $\mathrm{K}$ o qual chamaremos de anel dos inteiros de $\mathrm{K}$ e que pé seu único ideal maximal, ou seja, o anel $\mathscr{O}$ é local e $\mathscr{O}-\mathfrak{p}=\mathscr{O}^{\times}$, onde $\mathscr{O}^{\times}=\{x \in \mathscr{O}: x$ é invertível $\}$. Veja [6], Proposição 2.4.1.

$\mathrm{O}$ anel quociente $\mathscr{K}=\mathscr{O} / \mathfrak{p}$ é um corpo chamado de corpo residual. Ainda, os elementos de $\mathrm{K}$ que tem valor absoluto igual a 1 são chamados de unidades. Tais elementos formam um grupo multiplicativo, o grupo das unidades, que denotaremos por $\mathscr{O}^{*}=\{x \in K:|x|=1\}$. Definimos também o grupo de valores como sendo o subgrupo multiplicativo dos reais positivos $\mathscr{V}=\{|x|$ : $\left.x \in K^{*}\right\}$, onde $K^{*}=K-\{0\}$.

Definição 2.5.1 Dizemos que um valor absoluto não-arquimediano || é discreto, se o grupo de valores $\mathscr{V}$ for um subgrupo discreto de $\mathbb{R}_{+}^{*}$. Isto é, se existe um $\delta>0$ fixo tal que $1-\delta<|x|<1+\delta$, então $|x|=1$. 
Definição 2.5.2 Um corpo K com um valor absoluto || é dito corpo local se o par $(K, \mid$ |) satisfazer as seguintes condições:

1. || é não-arquimediano, discreto e não-trivial;

2. $K$ é completo relativo a || ;

3. O corpo residual de || é finito.

Exemplos típicos de corpos locais são os corpos p-ádicos $\mathbb{Q}_{p}$ e o corpo $F((x))$ das séries formais de Laurent em uma indeterminada sobre um corpo finito $\mathrm{F}$.

Definição 2.5.3 i) Seja K um corpo com valor absoluto || e topologia induzida por ||. Então uma cobertura aberta para $K$ é uma coleção de abertos de K cuja reunião é K. Se uma subcoleção de uma cobertura $C$ de $K$ ainda for uma cobertura de $K$, então dizemos que tal subcoleção é uma subcobertura de $K$.

ii) $K$ é dito compacto se e somente se qualquer cobertura aberta de $K$ admitir uma subcobertura finita.

iii) Um corpo normado(com norma igual ao seu valor absoluto) é dito localmente compacto se todo ponto de $K$ admitir uma vizinhança compacta. Entende-se por vizinhança compacta de um ponto q de K, um compacto contendo q e contido em algum aberto também contendo $q$.

Com estas definições, temos o seguinte resultado:

Teorema 2.5.1 Seja K um corpo com um valor absoluto não-arquimediano e discreto ||. Suponha que o corpo residual $\mathscr{O} / \mathfrak{p}$ de $K$ seja finito e que $K$ seja completo com respeito à || . Então o anel dos inteiros:

$$
\mathscr{O}=\bar{B}(0,1)=\{x \in K:|x| \leq 1\} \subset K
$$

é compacto na topologia induzida por ||. Ainda, Ké localmente compacto.

Demonstração: Ver [1], Teorema e Corolário, pg. 49 - 50.

Observação 2.5.1 A "recíproca” do Teorema anterior também é verdade, ou seja, se K for um corpo localmente compacto com respeito à um valor absoluto não-arquimediano ||, então: 
1. Ké completo;

2. o corpo residual $\mathscr{O} / \mathfrak{p}$ é finito;

3. o valor absoluto ||é discreto.

Para mais detalhes, ver [1], pg. 50. 


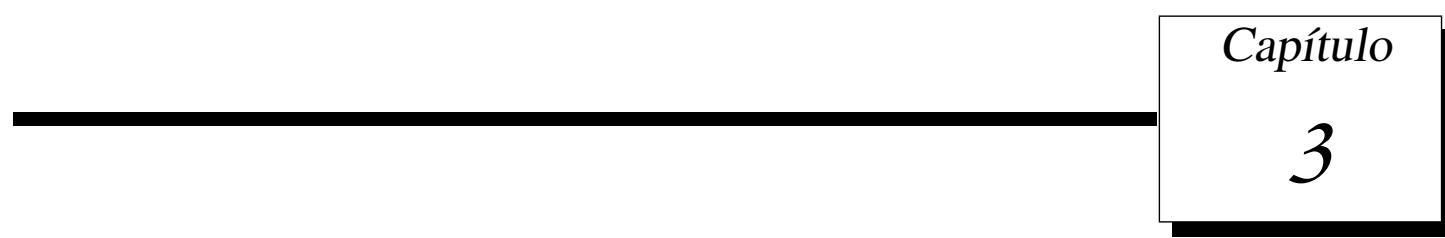

\section{Prova do Teorema de Tits}

Voltemos agora para a prova do Teorema de Tit's, que será feita através de 6 passos. Vamos mostrar que um grupo linear finitamente gerado o qual não é solúvel por finito, deve conter um subgrupo livre de posto 2. Esses passos nos darão uma prova completa do Teorema da Alternativa de Tits. Para compreensão da abordagem, é útil observarmos que, se G é um grupo com um subgrupo normal N, e dois elementos $a N$ e $b N$ de $\frac{G}{N}$ geram um subgrupo livre de posto 2 em $\frac{G}{N}$, então o subgrupo $\langle a, b\rangle$, gerado por $a$ e $b$ em $\mathrm{G}$ é livre de posto 2 .

Assim, afim de mostrarmos que $\mathrm{G}$ possui um subgrupo livre de posto 2, podemos passar para imagens homomorficas de G.

\section{Passo 1 - Redução ao caso irredutível}

Lema 3.0.1 Sejam $G_{1}$ e $G_{2}$ grupos.

1. Se $G_{1}$ e $G_{2}$ são solúveis por finito, então $G_{1} \times G_{2}$ é solúvel por finito;

2. Seja $H$ um subgrupo de $G_{1}$. Se $G_{1}$ é solúvel por finito, então $H$ é solúvel por finito;

3. Seja $H$ um subgrupo normal de $G_{1}$. Se $H$ é solúvel e $\frac{G_{1}}{H}$ é solúvel por finito, então $G_{1} e ́$ solúvel por finito.

Demonstração: 1 . Como por hipótese $G_{1}$ e $G_{2}$ são solúveis por finito, existem $H_{1} \triangleleft G_{1}$ e $H_{2} \triangleleft G_{2}$, $H_{1}, H_{2}$ solúveis com $\left[G_{1}: H_{1}\right]<\infty$ e $\left[G_{2}: H_{2}\right]<\infty$.

Note que $H_{1} \times H_{2} \triangleleft G_{1} \times G_{2}$. Como $H_{1}$ e $H_{2}$ são solúveis, $H_{1} \times H_{2}$ é solúvel e

$$
\frac{G_{1} \times G_{2}}{H_{1} \times H_{2}} \cong \frac{G_{1}}{H_{1}} \times \frac{G_{2}}{H_{2}}
$$


Desse modo, $\left[G_{1} \times G_{2}: H_{1} \times H_{2}\right]<\infty$ e portanto $G_{1} \times G_{2}$ é solúvel por finito.

2. Seja $H_{1} \triangleleft G_{1}$ solúvel tal que $\left[G_{1}: H_{1}\right]<\infty$. Considere o subgrupo $H \cap H_{1}$ de $H$. Note que $H \cap H_{1} \triangleleft H$ e $\left[H: H \cap H_{1}\right] \leq\left[G_{1}: H_{1}\right]<\infty$. Por outro lado, $H \cap H_{1}$ é solúvel, pois $H \cap H_{1}<H_{1}$ e $H_{1}$ é solúvel. Portanto $\mathrm{H}$ é solúvel por finito.

3. Como por hipótese $\frac{G}{H}$ é solúvel por finito, existe $\frac{L}{H} \triangleleft \frac{G}{H}$ solúvel tal que $\left[\frac{G}{H}: \frac{L}{H}\right]<\infty$. Assim, $L \triangleleft G$ e tem índice finito em G. Ainda, $\frac{L}{H}$ é solúvel e H é solúvel por hipótese. Logo L é solúvel e portanto G é solúvel por finito.

Seja $G \leq G L(V)$ finitamente gerado, onde V é um espaço vetorial de dimensão $n$ sobre um corpo $F$. Seja ainda $V=V_{0} \supset V_{1} \supset \cdots \supset V_{t}=\{0\}$ uma série de composição de comprimento $t \leq n$, ou seja, os espaços $\frac{V_{i-1}}{V_{i}}$ são irredutíveis, $i=1, \ldots, t$. Então $\mathrm{G}$ age naturalmente em cada $\frac{V_{i-1}}{V_{i}}$, $i=1, \ldots, t$.

Denote por $G_{i}=\operatorname{Im}\left(\rho_{i}\right) \leq G L\left(\frac{V_{i-1}}{V_{i}}\right)$, onde para cada $i=1, \ldots, t$ :

$$
\begin{aligned}
\rho_{i}: G & \longrightarrow G L\left(\frac{V_{i-1}}{V_{i}}\right) \\
x & \longmapsto \rho_{i}(x)
\end{aligned}
$$

é a ação natural.

Afirmamos que se cada $G_{i}, i=1, \ldots, t$ for solúvel por finito, então $\mathrm{G}$ também será solúvel por finito. Com efeito, façamos o caso $V \supset W \supset\{0\}$, isto é, o caso $t=2$. O caso geral segue por indução em $t$.

Sejam $\rho_{1}: G \longrightarrow G L\left(\frac{V}{W}\right)$ e $\rho_{2}: G \longrightarrow G L(W)$ as ações naturais de $G$ em $G L\left(\frac{V}{W}\right)$ e $G L(W)$, respectivamente. Dai temos $G_{1}=\operatorname{Im}\left(\rho_{1}\right) \leq G L\left(\frac{V}{W}\right)$ e $G_{2}=\operatorname{Im}\left(\rho_{2}\right) \leq G L(W)$. Sejam $A=\operatorname{Ker}\left(\rho_{1}\right)$ e $B=\operatorname{Ker}\left(\rho_{2}\right)$. Então $\frac{G}{A} \cong G_{1}$ e $\frac{G}{B} \cong G_{2}$.

Considere o homomorfismo:

$$
\begin{aligned}
\rho_{1} \times \rho_{2}: G & \longrightarrow G_{1} \times G_{2} \\
x & \longmapsto\left(\rho_{1}(x), \rho_{2}(x)\right) .
\end{aligned}
$$

Como estamos supondo $G_{1}$ e $G_{2}$ solúveis por finito, segue pelo item 1 do Lema anterior que $G_{1} \times G_{2}$ é solúvel por finito. Note que $\operatorname{Ker}\left(\rho_{1} \times \rho_{2}\right)=A \cap B$. $\operatorname{Logo}, \frac{G}{A \cap B} \cong \operatorname{Im}\left(\rho_{1} \times \rho_{2}\right)$ e pelo item 2 do Lema anterior, $\frac{G}{A \cap B}$ é solúvel por finito. Por outro lado, $A \triangleleft G$ e $B \triangleleft G$ implica que $A \cap B \triangleleft G$. Seja agora $x \in A \cap B$. Observe que $V \cong W \oplus \frac{V}{W}$. Assim, sendo $\left\{w_{1}, w_{2}, \ldots, w_{s}\right\}$ uma base de $\mathrm{W}$, podemos encontrar uma base $\overline{w_{s+1}}, \ldots, \overline{w_{n}}$ de $\frac{V}{W}$ tal que $\left\{w_{1}, w_{2}, \ldots, w_{s}, w_{s+1}, \ldots, w_{n}\right\}$ é base de V. 
Escrevendo $x \in A \cap B=\operatorname{Ker}\left(\rho_{1}\right) \cap \operatorname{Ker}\left(\rho_{2}\right)$ nesta base, obtemos que $x$ é da forma:

$$
x=\left(\begin{array}{ccccccccc}
1 & 0 & 0 & \cdots & 0 & * & * & \cdots & * \\
0 & 1 & 0 & \cdots & 0 & \vdots & \vdots & \cdots & * \\
0 & 0 & 1 & \cdots & 0 & * & * & \cdots & * \\
\vdots & \vdots & & \ddots & \vdots & \vdots & & & \vdots \\
0 & 0 & \cdots & 0 & 1 & * & \cdots & * & * \\
0 & 0 & 0 & \cdots & 0 & 1 & & & 0 \\
0 & \vdots & & & \vdots & & \ddots & & \vdots \\
\vdots & 0 & & & 0 & & & 1 & 0 \\
0 & 0 & \cdots & 0 & 0 & 0 & 0 & \cdots & 1
\end{array}\right) \in \operatorname{STL}(n)
$$

Como $x$ foi tomado arbitrariamente em $A \cap B$, segue que $A \cap B<S T L(n)$, sendo este solúvel pelo Lema 1.3.1. Logo, $A \cap B$ é solúvel. Desse modo, temos $A \cap B \triangleleft G, A \cap B$ solúvel e $\frac{G}{A \cap B}$ é solúvel por finito. Portanto pelo item 3 do Lema anterior, segue que G é solúvel por finito, como queríamos.

Assim, ao supormos que $\mathrm{G}$ não é solúvel por finito, concluimos que pelo menos algum $G_{i}(i=$ $1, \ldots, t)$ não é solúvel por finito. Logo, é suficiente mostrarmos que um tal $G_{i}$ contém um subgrupo livre de posto 2, pois $G_{i}$ é uma imagem homomórfica de $\mathrm{G}$.

Em outras palavras, substituindo $\mathrm{G}$ por $G_{i}$ e V por $\frac{V_{i-1}}{V_{i}}$, podemos supor que G é irredutível.

\section{Passo 2 - Restrição à componente conexa da identidade de $G$}

Seja $G^{0}$ a componente conexa da identidade de $G$. Sabemos que $G^{0}$ é um conexo na topologia de Zariski. Ainda, pelo Lema 1.1.1, $G^{0}$ é um subgrupo normal de $G$ e $\left[G: G^{0}\right]<\infty$.

Afirmamos que se $G$ não é solúvel por finito, então $G^{0}$ também não é solúvel por finito. Caso contrário, existiria $H \triangleleft G^{0}$, H solúvel tal que $\left[G^{0}: H\right]<\infty$. Logo $[G: H]<\infty$. Sendo $C=\{$ classes laterais à esquerda de $\mathrm{Hem} \mathrm{G}\}=\{a H \mid a \in G\}$, considere a aplicação:

$$
\begin{aligned}
& T: G \longrightarrow \mathscr{P}(C) \\
& g \longmapsto T_{g}: C \rightarrow C \\
& a H \mapsto g a H .
\end{aligned}
$$

É fácil ver que $T_{g}$ é uma bijeção e portanto $T$ está bem definida. Note que $\operatorname{Ker} T=\left\{g \in G \mid a g a^{-1} \in\right.$ $H, \forall a \in G\} \subseteq H$. Desse modo, temos que $K=\operatorname{Ker} T \triangleleft G, K$ tem índice finito em $G$ e é solúvel pois $K$ é subgrupo de $H$. Portanto $G$ é solúvel por finito, o que é um absurdo.

Diante disso, observe que como $G^{0}$ é subgrupo de G, é suficiente provarmos que $G^{0}$ contém um subgrupo livre de posto 2 . 


\section{Passo 3 - Escolhendo $x \in G$ de modo especial}

Definição 3.0.4 Seja G um grupo. Dizemos que Gé metabeliano se G possui um subgrupo normal abeliano $H$, cujo quociente $\frac{G}{H}$ é abeliano.

Lema 3.0.2 Seja G um grupo (não necessariamente linear). Se $G^{\prime}$ é finito, então G é solúvel por finito.

Demonstração: Seja $C=C_{G}\left(G^{\prime}\right) \leq G$ o centralizador de $G^{\prime}$ em $G$. Como $G^{\prime} \triangleleft G, \frac{C}{C \cap G^{\prime}} \cong \frac{C G^{\prime}}{G^{\prime}} \leq \frac{G}{G^{\prime}}$ e $\frac{G}{G^{\prime}}$ é abeliano, segue que $\frac{C}{C \cap G^{\prime}}$ é abeliano. Por outro lado $C \cap G^{\prime} \triangleleft C$ e é abeliano, o que implica que $\mathrm{C}$ é metabeliano. Em particular $\mathrm{C}$ é solúvel.

Considere o homomorfismo:

$$
\begin{aligned}
\varphi: G & \longrightarrow A u t\left(G^{\prime}\right) \\
g \longmapsto I_{g}: G^{\prime} & \rightarrow G^{\prime} \\
w & \mapsto g w g^{-1} .
\end{aligned}
$$

Note que $\operatorname{ker}(\varphi)=\left\{g \in G: g w=w g, \forall w \in G^{\prime}\right\}=C_{G}\left(G^{\prime}\right)=C$. Em particular $C \triangleleft G$. Pelo Teorema dos isomorfismos temos que:

$$
\frac{G}{C} \simeq \text { algum subgrupo de } \operatorname{Aut}\left(G^{\prime}\right)
$$

Assim, como por hipótese $G^{\prime}$ é finito, temos que $A u t\left(G^{\prime}\right)$ é finito o que implica que $\mathrm{C}$ tem índice finito em G. Portanto G é solúvel por finito.

Observação 3.0.2 Seja $\varphi: R \longrightarrow S$ um homomorfismo de anéis. Para cada inteiro $n \geq 1$, $\varphi$ induz um homomorfismo de anéis:

$$
\begin{gathered}
\phi: M(n, R) \longrightarrow M(n, S) \\
\left(\xi_{i j}\right) \longmapsto\left[\varphi\left(\xi_{i j}\right)\right] .
\end{gathered}
$$

Ainda, para qualquer subgrupo $G$ de $G L(n, R)$, $\phi$ nos dá um homomorfismo de grupos $\phi^{*}$ :

$$
\begin{aligned}
\phi^{*}: G & \longrightarrow G L(n, S) \\
A=\left(a_{i j}\right) & \longmapsto \phi^{*}(A)=\left[\varphi\left(a_{i j}\right)\right] .
\end{aligned}
$$


Chamamos $\phi^{*}$ de homomorfismo induzido em $G$ por $\varphi$. Note que se $\varphi$ for injetora, então $\phi^{*}$ também será.

Seja então E uma extensão de corpos de grau d sobre um corpo F. Logo, Eé um espaço vetorial de dimensão d sobre $F$ e existe um isomorfismo:

$$
\begin{gathered}
E \longrightarrow \bar{E} \subseteq \operatorname{Hom}_{F}(E, E) \\
x \longmapsto \quad \bar{x}: E \longrightarrow E \\
u \longmapsto u x
\end{gathered}
$$

Escolhendo uma base para E sobre F, temos então um homomorfismo injetor:

$$
\varphi: E \longrightarrow M(d, F)
$$

Assim, para cada subgrupo $G$ de $G L(n, E)$, temos o isomorfismo induzido:

$$
\varphi^{*}: G \longrightarrow \bar{G} \subseteq G L(n, M(d, F))
$$

Mas $G L(n, M(d, F))=G L(n d, F)$, e dai $G$ pode ser visto como um subgrupo de $G L(n d, F)$.

Proposição 3.0.1 Seja G um grupo linear finitamente gerado e H um subgrupo de G. Se cada $x \in H$ tem todos os seus autovalores raizes da unidade, então $H$ tem expoente finito ( isto é, existe $e \in \mathbb{N}$ tal que $x^{e}=I d$, para todo $x \in H$ ).

Demonstração: Temos por hipótese que $\mathrm{G}$ é finitamente gerado. Sejam $x_{1}, \ldots, x_{r}$ as matrizes geradoras de $\mathrm{G}, F_{1}$ o subcorpo de $\mathrm{F}$ gerado pelas entradas dessas matrizes e $F_{0}$ o subcorpo primo de F. Então $G \leq G L\left(n, F_{1}\right)$. Por outro lado, como $F_{1}$ é finitamente gerado, segue que existe uma base de transcendência finita $\left\{t_{1}, \ldots, t_{k}\right\}$ de $F_{1}$ sobre $F_{0}$, ou seja, um conjunto maximal de elementos algebricamente independentes sobre $F_{0}$.

Seja $E=F_{0}\left(t_{1}, \ldots, t_{k}\right)$. Então a extensão $E \subseteq F_{1}$ é algébrica finita, digamos de grau d.

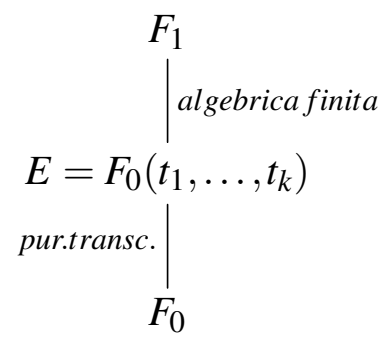

Assim, pela Observação 3.0.2, G é isomorfo a um subgrupo $\bar{G}$ de $G L(n d, E)$.

Para cada $x \in H$, seja $f_{x}(t) \in E[t]$ o polinômio minimal de $x$ sobre E. Agora como por hipótese 
$x$ possui todos seus autovalores raízes da unidade, temos que o polinômio característico $p_{x}(t)$ de $x$ tem todas as suas raízes sendo raízes da unidade. Logo, todas as raizes de $f_{x}(t)$ também são raizes da unidade e como os coeficientes de $f_{x}(t)$ são somas de produtos de raízes da unidade eles são algébricos sobre $F_{0}$. Sendo a extensão $E / F_{0}$ puramente transcendente, os únicos elementos algébricos de E sobre $F_{0}$ estão em $F_{0}$. Assim $f_{x}(t) \in F_{0}[t]$

Temos dois casos:

Caso $1(\operatorname{car} F=0)$. Nesse caso $F_{0}=\mathbb{Q}$. Considere agora o polinômio:

$$
(t+1)^{n d}=t^{n d}+\left(\begin{array}{c}
n d \\
1
\end{array}\right) t^{n d-1}+\left(\begin{array}{c}
n d \\
2
\end{array}\right) t^{n d-2}+\cdots+\left(\begin{array}{c}
n d \\
n d-1
\end{array}\right) t+1
$$

Para cada $x \in H, p_{x}(t)$ é da forma:

$$
p_{x}(t)=\left(t-\xi_{1}\right) \ldots\left(t-\xi_{n d}\right)=t^{n d}+\underbrace{\left(\xi_{1}+\cdots+\xi_{n d}\right)}_{\left(\begin{array}{c}
n d \\
1
\end{array}\right) \text { somas }} t^{n d-1}+\underbrace{\left(\xi_{1} \xi_{2}+\xi_{1} \xi_{3}+\cdots\right)}_{\left(\begin{array}{c}
n d \\
2
\end{array}\right) \text { somas }} t^{n d-2}+\cdots
$$

Como os $\xi_{i}^{\prime} s \in \mathbb{C}$ são raízes da unidade, temos que $\left|\xi_{1}\right|=\cdots=\left|\xi_{n d}\right|=1, i=1, \ldots, n d$. Daí:

$$
\begin{gathered}
\left|\xi_{1}+\cdots+\xi_{n d}\right| \leq n d \\
\left|\xi_{1} \xi_{2}+\xi_{1} \xi_{3}+\cdots+\xi_{n d-1} \xi_{n d}\right| \leq\left(\begin{array}{c}
n d \\
2
\end{array}\right)
\end{gathered}
$$

que são exatamente os coeficientes de $(t+1)^{n d}$. Por outro lado, $f_{x}(t) \mid p_{x}(t)$. Logo, os coeficientes de $f_{x}(t)$ são dominados pelos coeficientes de $(t+1)^{n d}$. Desse modo, concluímos que existem apenas um número finito de $f_{x}(t)$, quando $x$ percorre $H$ e como $x$ e $y$ possuem a mesma ordem quando $f_{x}(t)=f_{y}(t), x, y \in H$, temos que $\mathrm{H}$ tem expoente finito, ou seja, existe um $N \in \mathbb{N}$ tal que $x^{N}=I d_{n d}$. Em outras palavras, $m(x) \leq N, \forall x \in G$. Em particular, existem apenas um número finito de raizes da unidade que são autovalores de $x$, para todo $x \in H$.

Caso $2(\operatorname{car} F=p>0)$. Nesse caso $F_{0}$ tem $\mathrm{p}$ elementos e, como $f_{x}(t) \in F_{0}[t]$, temos apenas um número finito de polinômios minimais $f_{x}(t)$ quando $x$ percorre $\mathrm{H}$. Como no primeiro caso, $\mathrm{H}$ tem expoente finito.

Uma propriedade bastante importante sobre grupos lineares é que (ao contrário de grupos mais gerais), se um grupo linear tem expoente finito ele tende a ser finito. De fato, como nos mostra 
o teorema abaixo, se a caraterística do corpo de base for zero isso é verdade. Se a característica for positiva precisamos de hipóteses extras. A prova do teorema abaixo está fora do alcance dessa dissertacão. Assim vamos apenas enunciá-lo e dar uma referência.

Teorema 3.0.2 Seja $G \leq G L(n, F)$ um grupo linear. Suponha que G tem expoente finito e. Então:

1. Se carF $=0$, então $G$ é finito $e|G| \leq e^{n^{3}}$.

2. Se carF $=p>0$, então $G$ possui um $p$-subgrupo nilpotente e normal $N$, que consiste apenas de elementos nilpotentes, tal que $[G: N] \leq e^{n^{3}}$. Em particular, $N=\{1\}$ (e G é finito) se $p \nmid n$ ou se $G$ é completamente redutível.

Demonstração: Ver Teorema 2.9, [2],pg.40

Corolário 3.0.1 Seja G um grupo linear finitamente gerado e completamente redutível sobre um corpo F. Se $G$ não é solúvel por finito, então existe $x \in G^{\prime}$ com um autovalor que não é raiz da unidade.

Demonstração: Se, para cada $x \in G^{\prime}$, todos seus autovalores são raízes da unidade, pela Proposição anterior $G^{\prime}$ tem expoente finito. Se $\operatorname{car} F=0, G^{\prime}$ é finito. Se $c a r F=p>0$, sendo G completamente redutível e $G^{\prime}$ normal em G, pelo Teorema de Clifford (Teorema 2.2, [2], pg.25 ), $G^{\prime}$ é completamente redutível e pelo Teorema anterior $G^{\prime}$ é finito. Em ambos os casos $\mathrm{G}$ é solúvel por finito pelo Lema 3.0.2.

\section{Passo 4 - Extensão de F a um corpo normado localmente compacto}

Neste passo, iremos construir um corpo normado localmente compacto no qual G tem uma representação. Seja $E_{0}$ o subcorpo primo de $\mathrm{F}$ e seja $\mathrm{E}$ o subcorpo de $\mathrm{F}$ gerado pelas entradas de um conjunto de matrizes geradoras de G. Assim, $G \leq G L(n, E)$ e E é finitamente gerado sobre $E_{0}$.

Seja $x \in G$ e $\xi$ um autovalor de $x$ tal que $\xi$ não é raiz da unidade, o qual existe pelo passo 3. $\mathrm{O}$ próximo resultado irá mostrar como podemos estender E para um corpo normado localmente compacto $(K,||)$ tal que $|\xi| \neq 1$. Usaremos alguns resultados do capítulo anterior e de [3] - Capítulo V.

Proposição 3.0.2 Nas condições acima, supondo que $\xi \in E$ não seja uma raiz da unidade, existe uma extensão $K / E$ tal que $(K,||)$ é um corpo normado localmente compacto satisfazendo $|\xi| \neq 1$. 
Demonstração: Como E é finitamente gerado sobre $E_{0}$ segue que existe uma base de transcendência finita $T=\left\{t_{1}, \ldots, t_{k}\right\}$ de E sobre $E_{0}$ e E é de grau finito sobre $E_{0}(T)$. Temos:

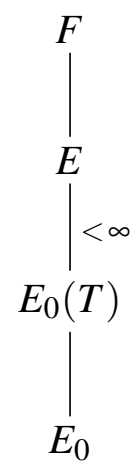

Temos dois casos:

1ํㅡㄹ caso: $\operatorname{car} E=p>0$. Primeiro note que como por hipótese $\xi$ não é raiz da unidade, então $\xi$ é transcendente sobre o corpo primo $G F(p)$. Seja $P((t))$ o corpo das séries formais de Laurent. Considere então a inclusão:

$$
\begin{gathered}
\varphi: G F(p)(\xi) \longrightarrow P((t)) \\
\xi \longmapsto t
\end{gathered}
$$

Como o grau de transcendência de $P((t))$ sobre $G F(p)(\xi)$ é infinito, esta inclusão pode ser estendida a uma inclusão $\varphi_{1}: E \longrightarrow K$, onde K é uma extensão finita de $P((t))$.

$2^{\underline{0}}$ caso: $\operatorname{car} E=0$, isto é, $E_{0}=\mathbb{Q}$. Vamos mostrar que existe uma imersão $\phi$ de $\mathrm{E}$ em um corpo normado localmente compacto $(L,||) \operatorname{com}|\phi(\xi)| \neq 1$. Isto nos dará uma extensão $K \simeq L$ de $\mathrm{E}$ com as propriedades requeridas. Com efeito, temos duas situações:

Se $\xi$ for transcendente sobre $\mathbb{Q}$, como no caso anterior podemos tomar $\xi \in T$. Ainda, como $\mathbb{C}$ tem grau de transcendência infinito sobre $\mathbb{Q}$, segue que existe uma imersão $\phi_{0}: \mathbb{Q}(T) \longrightarrow \mathbb{C}$ tal que $\phi_{0}(\xi)$ é um elemento transcendente em $\mathbb{C}$ de valor absoluto $>1$. Como $\mathbb{C}$ é algebricamente fechado e $[E: \mathbb{Q}(T)]<\infty$, segue pelo Teorema 2.4.6 que $\phi_{0}$ pode ser estendida a uma imersão

$$
\phi: E \longrightarrow \mathbb{C}
$$

Mas $\mathbb{C}$ é localmente compacto (com a norma usual) e $|\xi|=|\phi(\xi)|=\left|\phi_{0}(\xi)\right|>1$. Tomando $L=\mathbb{C}$, temos as condições desejadas.

Se $\xi$ for algébrico sobre $\mathbb{Q}$, então considere $m(X)=q_{0}+q_{1} x+\cdots+q_{n-1} x^{d-1}+x^{d}$ o polinômio minimal de $\xi$ sobre $\mathbb{Q}$, onde $q_{i}=\frac{a_{i}}{b_{i}}, a_{i}, b_{i} \in \mathbb{Z}, b_{i} \neq 0,0 \leq i \leq d-1$. Assim temos novamente duas situações a analisar. Se todos os coeficientes de $m(X)$ forem inteiros, isto é, $\xi$ é um inteiro 
algébrico, então $m(X)$ possui uma raiz $\eta \in \mathbb{C}$ de norma estritamente maior do que 1 . De fato, suponha que $\left|\alpha_{i}\right| \leq 1, i=1, \ldots, d$, onde $\left\{\alpha_{1}, \ldots, \alpha_{d}\right\}$ são as raizes de $m(X)$. Se existir $\alpha_{i}$ raiz de $m(X)$ tal que $\left|\alpha_{i}\right|<1$, então considerando o coeficiente $q_{0}=(-1)^{d} \alpha_{1} \ldots \alpha_{d} \in \mathbb{Z}$, temos que $\left|q_{0}\right|=\left|\alpha_{1} \ldots \alpha_{d}\right|<1$, o que implica $q_{0}=0$. Mas isto é um absurdo pois $m(X)$ é irredutível. Assim, $\left|\alpha_{i}\right|=1$ para toda raiz $\alpha_{i}$ de $m(X)$. Logo, pelo Teorema de Kronecker (Teorema 2.4.5), $\xi$ é uma raiz da unidade, o que nos leva novamente a uma contradição. Portanto $m(X)$ possui ao menos uma raiz, digamos $\eta \in \mathbb{C}$, de norma estritamente maior do que 1 . Considere então a $\mathbb{Q}$-imersão:

$$
\begin{aligned}
\phi_{0}: \mathbb{Q}(\xi) & \longrightarrow \mathbb{Q}(\eta) \subset \mathbb{C} \\
\xi & \longmapsto \eta
\end{aligned}
$$

Seja $T_{1}$ uma base de transcendência de $\mathrm{E}$ sobre $\mathbb{Q}(\xi)$. Então podemos estender $\phi_{0}$ a uma imersão:

$$
\begin{aligned}
\phi_{1}: \mathbb{Q}\left(T_{1} \cup\{\xi\}\right) & \longrightarrow \mathbb{C} \\
\xi & \longmapsto \eta
\end{aligned}
$$

Como a extensão $\mathbb{Q}\left(T_{1} \cup\{\xi\}\right) \subset E$ é algébrica finita e $\mathbb{C}$ é algebricamente fechado, segue do Teorema 2.4.6 que $\phi_{1}$ pode ser estendida a uma $\mathbb{Q}$ - imersão

$$
\phi: E \longrightarrow \mathbb{C}
$$

Novamente, como $\mathbb{C}$ é localmente compacto (com a norma usual) e $|\phi(\xi)|=|\eta|>1$, tomando $L=\mathbb{C}$, segue o resultado para o caso em que $m(X)$ possui todos os seus coeficientes inteiros.

Suponha agora que $m(X)$ tenha algum coeficiente $q_{j}=\frac{a_{j}}{b_{j}}$ não inteiro, com $m d c\left(a_{j}, b_{j}\right)=1$. Seja $\mathrm{p}$ um primo que divide $b_{j}$, e considere $\left(\mathbb{Q}_{p},||_{0}\right)$ o completamento p-ádico dos racionais. Sejam ainda $L_{1}$ o corpo de fatoração de $m(X)$ sobre $\mathbb{Q}_{p} \mathrm{e}||_{1}$ a (única) extensão da norma ||$_{0}$ para $L_{1}$, a qual existe e é não arquimediana pelo Teorema 2.4.1.

Note que ao menos uma das funções simétricas elementares das raízes de $m(X)$ tem norma estritamente maior do que 1 , pois considere justamente a função simétrica elementar referente ao coeficiente não inteiro $q_{j}$. Temos:

$$
q_{j}=(-1)^{j}\left(\alpha_{1} \ldots \alpha_{j}+\alpha_{1} \ldots \alpha_{j-1} \alpha_{j+1}+\cdots+\alpha_{1} \ldots \alpha_{j-1} \alpha_{d}+\cdots+\alpha_{d-j} \ldots \alpha_{d}\right)
$$

Como $p \mid b_{j}$, segue que $b_{j}=p^{l} . c_{j}$, com $c_{j}, l \in \mathbb{Z}, l \geq 1$. Logo, $q_{j}=\frac{a_{j}}{p^{l} c_{j}}$ e assim $\left|q_{j}\right|_{0}=2^{l}>1$. Consequentemente, $|\eta|_{1}>1$ para ao menos uma raiz $\eta$ de $m(X)$ ( onde $\eta$ é um dos $\alpha_{i}^{\prime} s$ ), pois sendo $\left|q_{j}\right|_{0}>1$, então

$$
1<\left|q_{j}\right|_{0}=\left|\alpha_{1} \ldots \alpha_{j}+\alpha_{1} \ldots \alpha_{j-1} \alpha_{j+1}+\cdots+\alpha_{1} \ldots \alpha_{j-1} \alpha_{d}+\cdots+\alpha_{d-j} \ldots \alpha_{d}\right|_{0} \leq
$$




$$
\leq \max \left\{\left|\alpha_{1} \ldots \alpha_{j}\right|_{1},\left|\alpha_{1} \ldots \alpha_{j-1} \alpha_{j+1}\right|_{1}, \ldots,\left|\alpha_{1} \ldots \alpha_{j-1} \alpha_{d}\right|_{1}, \ldots,\left|\alpha_{d-j} \ldots \alpha_{d}\right|_{1}\right\}
$$

Neste caso, considere então a $\mathbb{Q}$ - imersão:

$$
\begin{aligned}
\phi_{0}: \mathbb{Q}(\xi) & \longrightarrow L_{1} \\
\xi & \longmapsto \eta
\end{aligned}
$$

Como $\mathbb{Q}_{p}$ é não enumerável, segue que $\mathbb{Q}_{p}$ tem grau de transcendência infinito sobre $\mathbb{Q}$. Consequentemente, $L_{1}$ também tem grau de transcendência infinito sobre $\mathbb{Q}$. Logo, sendo $T_{1}$ uma base de transcendência de $\mathrm{E}$ sobre $\mathbb{Q}(\xi)$, podemos estender $\phi_{0}$ a uma imersão:

$$
\begin{aligned}
\phi_{1}: \mathbb{Q}\left(T_{1} \cup\{\xi\}\right) & \longrightarrow L_{1} \\
\xi & \longmapsto \eta
\end{aligned}
$$

Logo, $\phi_{1}$ pode ser estendida a uma imersão $\phi: E \longrightarrow L$, onde L é alguma extensão finita de $L_{1}$ da seguinte maneira: seja $T_{1}^{\prime}=\phi_{1}(T)$. Então por meio de $\phi_{1}$, temos um isomorfismo:

$$
\phi_{1}: \mathbb{Q}(\xi)\left(T_{1}\right) \subset E \longrightarrow \mathbb{Q}(\eta)\left(T_{1}^{\prime}\right) \subset L_{1}
$$

Seja $L_{1}^{a}$ o fecho algébrico de $L_{1}$. Então como a extensão $\mathbb{Q}(\xi)\left(T_{1}\right) \subset E$ é algébrica finita e $L_{1}^{a} \supset L_{1}$ é algebricamente fechado, segue do Teorema 2.4.6 que $\phi_{1}$ se estende a uma imersão $\phi$ :

$$
\phi: E \longrightarrow L_{1}^{a}
$$

Agora, identificando E com sua imagem $\phi(E) \subseteq L_{1}^{a}$, temos $\left[\phi(E): \mathbb{Q}(\eta)\left(T_{1}^{\prime}\right)\right]<\infty$, pois $[E$ : $\left.\mathbb{Q}(\xi)\left(T_{1}\right)\right]<\infty$.

Note agora que $\phi(E)$ e $L_{1}$ são subcorpos de $L_{1}^{a}$. Tomando $L=\phi(E) L_{1}$, obtemos $\phi(E) \subset L$ e $L_{1} \subset L$. Temos o seguinte diagrama:

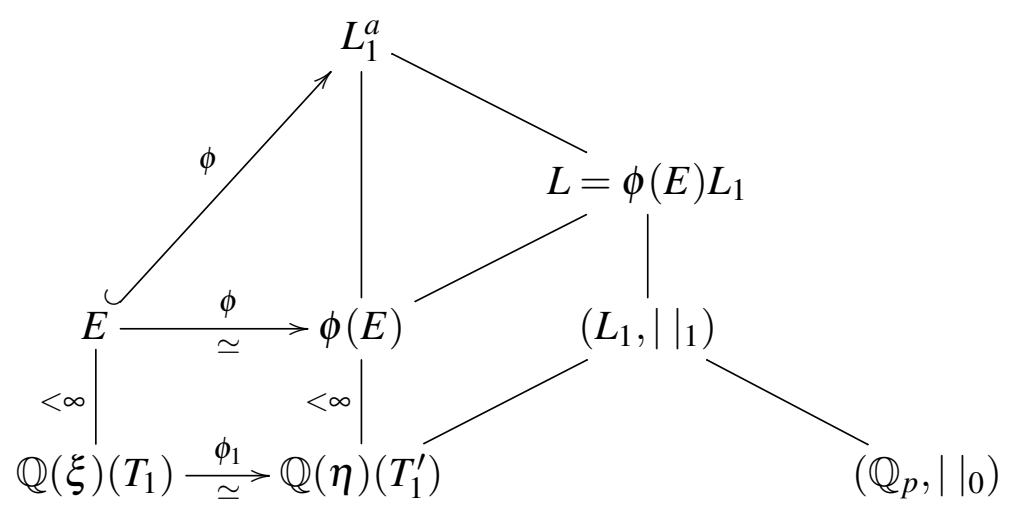


Agora como $\left[\phi(E): \mathbb{Q}(\eta)\left(T_{1}^{\prime}\right)\right]<\infty$, segue de [3] (Proposição $1.7-\operatorname{pg} .228$ ) que $\left[L: L_{1}\right]<\infty$ e portanto a norma em $L_{1}$ pode ser estendida unicamente para uma norma | | em L. O corpo normado Lé localmente compacto já que este é uma extensão finita do corpo localmente compacto $\left(\mathbb{Q}_{p},||_{0}\right)$. Por outro lado $|\phi(\xi)|=|\eta|>1$, e assim segue a extensão desejada de E a um corpo normado localmente compacto.

Podemos considerar agora $G<G L(n, K)$, $(K,||)$ corpo localmente compacto satisfazendo: existe $x \in G$ com um autovalor $\xi \in K$ tal que $|\xi| \neq 1$.

\section{Passo 5 - Mudança de representação}

$\mathrm{O}$ que faremos neste passo, é construir uma representação $\rho$ de $\mathrm{G}$, de modo que $\rho(x)$, onde x é o elemento considerado nos passos 3 e 4 , tenha um único autovalor simples de norma máxima. $\mathrm{O}$ próximo resultado nos garante que tal representação existe.

Proposição 3.0.3 Seja $(K,||)$ um corpo normado. Suponha que $G \leq G L(n, K)$ possua um elemento $x$ cujos autovalores $\lambda_{1}, \lambda_{2}, \ldots, \lambda_{n}$ pertençam a $K$ e satisfaçam

$$
\left|\lambda_{1}\right|=\left|\lambda_{2}\right|=\cdots=\left|\lambda_{r}\right|>\left|\lambda_{r+1}\right| \geq \cdots \geq\left|\lambda_{n}\right|
$$

Então existe uma representação $\rho: G \longrightarrow G L\left(\left(\begin{array}{c}n \\ r\end{array}\right), K\right)$ tal que $\rho(x)$ tem um único autovalor simples de norma máxima. Mais ainda, se $G$ for conexo na topologia de Zariski, então $\rho(G)$ também será.

Demonstração: Primeiro note que podemos assumir, sem perda de generalidade, (substituindo G por um grupo conjugado se necessário ), que $x$ está na sua forma de Jordan. Logo, sendo $\left\{e_{1}=(1,0, \ldots, 0), \ldots, e_{n}=(0,0, \ldots, 0,1)\right\}$ a base canônica de $K^{n}$, então para cada $i=1,2, \ldots, n$ temos $x e_{i}=\lambda_{i} e_{i}$ ou $x e_{i}=\lambda_{i} e_{i}+e_{i-1}$. Considere agora a álgebra exterior

$$
E=\bigwedge K^{n}=K \oplus K^{n} \oplus \wedge^{2} K^{n} \oplus \cdots \oplus \wedge^{n} K^{n}
$$

Se denotarmos por $e_{\Delta}=e_{i_{1}} \wedge e_{i_{2}} \wedge \cdots \wedge e_{i_{s}}$ para cada subconjunto $\Delta=\left\{i_{1}, i_{2}, \ldots, i_{s}\right\}$ de $\{1,2, \ldots, n\}$ com $i_{1}<i_{2}<\cdots<i_{s}$, então o conjunto de todos esses $e_{\Delta}$ 's formam uma K-base para E.

Note agora que $\mathrm{G}$ age linearmente em $\mathrm{E}$ de um modo natural:

$$
\phi: G \longrightarrow G L(E)
$$




$$
\begin{aligned}
& g \longmapsto \phi_{g}: E \longrightarrow E \\
& \underbrace{e_{i_{1}} \wedge \cdots \wedge e_{i_{s}}}_{e_{\Delta}} \longmapsto \phi_{g}\left(e_{\Delta}\right)=g e_{i_{1}} \wedge \cdots \wedge g e_{i_{s}}
\end{aligned}
$$

Seja V o subsespaço de E gerado pelos $e_{\Delta}$ 's tal que $|\Delta|=r$, isto é, $V=\bigwedge^{r} K^{n}$ e assim $\operatorname{dim}(V)=$ $\left(\begin{array}{l}n \\ r\end{array}\right)$. Ainda, pela ação $\phi$, temos que V é um subespaço G-invariante de E. Logo para cada $g \in G$, podemos restringir $\phi_{g}$ à $\mathrm{V}$ obtendo assim uma representação $\rho: G \longrightarrow G L\left(\left(\begin{array}{c}n \\ r\end{array}\right), K\right)$.

Vamos mostrar agora que $\rho(x)$ possui um único autovalor de norma máxima. De fato, seja $\mathrm{S}$ o conjunto formado pelos r-subconjuntos de $\{1,2, \ldots, n\}$. Definamos uma relação de ordem parcial $\leq$ em $\mathrm{S}$ do seguinte modo: se $\Delta=\left\{i_{1}, \ldots, i_{r}\right\}$ e $\Gamma=\left\{j_{1}, \ldots, j_{r}\right\}$ elementos de $\mathrm{S}$, então $\Delta \leq \Gamma$ se $i_{t} \leq j_{t}, \forall t$. Note que:

$$
\rho(x) e_{\Delta}=\lambda_{\Delta} e_{\Delta}+\sum \alpha_{\Gamma} e_{\Gamma}
$$

onde $\lambda_{\Delta}=\prod_{i \in \Delta} \lambda_{i}$, e a somatória em (1) é sobre $\Gamma \in S$ tais que $\Gamma<\Delta$ e $\alpha_{\Gamma} \in K$. Em particular, se $\Delta_{*}=\{1,2, \ldots, r\}$ então $\rho(x) e_{\Delta_{*}}=\lambda_{\Delta_{*}} e_{\Delta_{*}}$ já que não existe $\Gamma<\Delta_{*}$ em S. Sendo assim, $\lambda_{\Delta_{*}}$ é um autovalor de $\rho(x)$.

Suponha agora que $\mu \neq \lambda_{\Delta_{*}}$ seja um autovalor de $\rho(x)$ com um autovetor $v \in V$, onde $v$ não é um múltiplo escalar de $e_{\Delta_{*}}$. Escrevendo:

$$
v=\sum \beta_{\Gamma} e_{\Gamma}
$$

e escolhendo $\Delta$ maximal com respeito a ordem parcial em $\mathrm{S}$ tal que $\beta_{\Delta} \neq 0$ temos:

$$
\begin{gathered}
\rho(x) v=\rho(x)\left(\sum \beta_{\Gamma} e_{\Gamma}\right)=\rho(x)\left(\beta_{\Delta} e_{\Delta}+\sum_{\Gamma^{\prime}} \beta_{\Gamma^{\prime}} e_{\Gamma^{\prime}}\right)= \\
=\rho(x) \beta_{\Delta} e_{\Delta}+\rho(x) \sum_{\Gamma^{\prime}} \beta_{\Gamma^{\prime}} e_{\Gamma^{\prime}}=^{(1)} \beta_{\Delta} \lambda_{\Delta} e_{\Delta}+\underbrace{\sum_{T} \alpha_{T} e_{T}+\sum_{T^{\prime}} \beta_{T^{\prime}} e_{T^{\prime}}}_{R_{1}},
\end{gathered}
$$

sendo $\Gamma^{\prime}, T$, e $T^{\prime}$ elementos da ordem parcial $\leq$ comparáveis ou não com $\Delta$ e $\alpha_{T}$ e $\beta_{T^{\prime}}$ elementos de K.

Por outro lado:

$$
\mu v=\mu\left(\beta_{\Delta} e_{\Delta}+\sum_{\Gamma^{\prime}} \beta_{\Gamma^{\prime}} e_{\Gamma^{\prime}}\right)=\mu \beta_{\Delta} e_{\Delta}+\underbrace{\sum_{\Gamma^{\prime}} \mu \beta_{\Gamma^{\prime}} e_{\Gamma^{\prime}}}_{R_{2}}
$$


Agora como $\Delta$ foi tomado maximal em $\mathrm{S} \operatorname{com} \beta_{\Delta} \neq 0$ e $\rho(x) v=\mu \nu$, segue que:

$$
\beta_{\Delta} \lambda_{\Delta} e_{\Delta}+R_{1}=\mu \beta_{\Delta} e_{\Delta}+R_{2}
$$

o que implica $\beta_{\Delta} \lambda_{\Delta}=\mu \beta_{\Delta}$ e assim $\mu=\lambda_{\Delta}$. Logo, $|\mu|=\left|\lambda_{\Delta}\right|<\left|\lambda_{\Delta_{*}}\right|$, por hipótese. Assim $\rho(x)$ possui $\lambda_{\Delta_{*}}$ como seu único autovalor de norma máxima. Finalmente, sendo $\rho$ uma aplicação racional de $G \leq G L(n, K)$ em $G L\left(\left(\begin{array}{c}n \\ r\end{array}\right), K\right)$, então pela Observação 1.1.2, $\rho$ é contínua na topologia de Zariski. Portanto se $\mathrm{G}$ for conexo na topologia de Zariski, $\rho(G)$ também será.

\section{Passo 6 - Modificando o elemento $x$}

Temos $x \in G L(n, K)$. Os autovalores $\lambda_{1}, \lambda_{2}, \ldots \lambda_{n}$ de $x$ pertencem à uma extensão finita $K_{1}$ de $\mathrm{K}$. Lembrando que $(K,||)$ é localmente compacto, pela Observação 2.5.1 (K,| |) é completo e assim pelos Teoremas 2.4.2 e 2.4.3 existe uma única extensão ||$_{1}$ de || $\operatorname{para} K_{1}, \operatorname{com}\left|\lambda_{i}\right|_{1}(i=1,2, \ldots, n)$ independentes da escolha de $K_{1}$. Assim, trocando $(K,||)$ por $\left(K_{1},||_{1}\right)$ se necessário, podemos assumir que todos os autovalores de $x$ estão em K.

Definição 3.0.5 Diremos que $\lambda_{i}$ é um autovalor dominante para $x$ se $\left|\lambda_{i}\right|_{1}>\left|\lambda_{j}\right|_{1}$, para todo $j \neq i$.

Lembramos que pelo passo 5, temos uma representação $\rho: G \longrightarrow G L(n, K)$ tal que $\rho(x)$ tem autovalor dominante. Nosso objetivo nesse passo é mostrar que existe $x_{0} \in G$ tal que ambos $\rho\left(x_{0}\right)$ e $\rho\left(x_{0}\right)^{-1}$ possuem autovalores dominantes.

Lema 3.0.3 Seja F um corpo qualquer e $G \leq G L(m, F)$ um grupo linear irredutível. Sejam ainda $0 \neq v_{1} \in F^{m} e v_{2} \in F^{m}$. Então existe uma combinação $F$-linear $a=\alpha_{1} g_{1}+\cdots+\alpha_{t} g_{t}$, com $\alpha_{i} \in F$, $g_{i} \in G, i=1, \ldots, t$, tal que $a v_{1}=v_{2}$.

Demonstração: Considere o subespaço gerado por $G v_{1}$, isto é, o espaço:

$$
\left\langle G v_{1}\right\rangle=\left\{\lambda_{1} g_{1} v_{1}+\cdots+\lambda_{s} g_{s} v_{1} ; \lambda_{i} \in F, g_{i} \in G, s \geq 1\right\}
$$

Note que $\left\langle G v_{1}\right\rangle$ é G-invariante e é diferente de $\{0\}$ já que $g v_{1} \neq 0$, para todo $g \in G$. Logo, como $\mathrm{G}$ é irredutível, segue que $\left\langle G v_{1}\right\rangle=F^{m}$ e assim dado $v_{2} \in F^{m}$, existe $\alpha_{1} g_{1} v_{1}+\cdots+\alpha_{t} g_{t} v_{1} \in\left\langle G v_{1}\right\rangle$ tal que $\alpha_{1} g_{1} v_{1}+\cdots+\alpha_{t} g_{t} v_{1}=v_{2}$, ou seja, $\left(\alpha_{1} g_{1}+\cdots+\alpha_{t} g_{t}\right) v_{1}=v_{2}$. Portanto basta tomar $a=$ $\alpha_{1} g_{1}+\cdots+\alpha_{t} g_{t}$.

Proposição 3.0.4 Seja $(K,||)$ um corpo localmente compacto. Suponha que $G \leq G L(n, K)$ seja irredutível e conexo na topologia de Zariski. Se G possuir um elemento x cujos autovalores 
pertençam a $K$ e com um autovalor dominante, então $G$ conterá um elemento $x_{0}$ tal que ambos $x_{0}$ e $x_{0}^{-1}$ possuam autovalores dominantes.

Demonstração: Sejam $\lambda_{1}, \lambda_{2}, \ldots, \lambda_{n}$ os autovalores de $x$ com

$$
\left|\lambda_{1}\right|>\left|\lambda_{2}\right| \geq \cdots \geq\left|\lambda_{n}\right|
$$

Sem perda de generalidade, podemos assumir que $x$ está na forma de Jordan:

$$
x=\left[\begin{array}{cccc}
\lambda_{1} & 0 & \cdots & 0 \\
0 & \lambda_{2} & \cdots & 0 \\
\vdots & * & & \vdots \\
0 & * & * \cdots & \lambda_{n}
\end{array}\right]
$$

Afirmação: Se $c a r K=p>0$ então $x^{p^{N}}$ é diagonal, para algum $\mathrm{N}$ suficientemente grande. De fato, observe primeiro que o polinômio característico $p_{x}(t)$ de $x$ é da forma :

$$
p_{x}(t)=\left(t-\lambda_{1}\right)\left(t-\lambda_{2}\right)^{\alpha_{2}} \ldots\left(t-\lambda_{n}\right)^{\alpha_{n}}
$$

Logo, o polinômio minimal de $x$ é da forma

$$
m_{x}(t)=\left(t-\lambda_{1}\right)\left(t-\lambda_{2}\right)^{m_{2}} \ldots\left(t-\lambda_{n}\right)^{m_{n}}
$$

com $m_{i} \leq \alpha_{i}, i=2, \ldots, n$. Assim, o bloco de Jordan para o autovalor $\lambda_{1}$ de $x$ é o próprio $\lambda_{1}$ e para cada bloco de Jordan de $x$

$$
J_{x}\left(\lambda_{i}\right)=\left[\begin{array}{ccccc}
\lambda_{i} & 0 & 0 & \cdots & 0 \\
a_{i 1} & \lambda_{i} & 0 & \cdots & 0 \\
0 & a_{i 2} & \lambda_{i} & 0 \cdots & 0 \\
\vdots & 0 & \cdots & \vdots & \vdots \\
0 & 0 & \cdots & a_{i\left(n_{i}-1\right)} & \lambda_{i}
\end{array}\right]_{n_{i} \times n_{i}}
$$

onde $a_{i 1}, \ldots, a_{i\left(n_{i}-1\right)}, i=2, \ldots, n$ são $0^{\prime} s$ ou $1^{\prime} s$, temos $J_{x}\left(\lambda_{i}\right)=\lambda_{i} I_{n_{i}}+M_{n_{i}}$, onde

$$
M_{n_{i}}=\left[\begin{array}{ccccc}
0 & 0 & 0 & \cdots & 0 \\
a_{i 1} & 0 & 0 & \ldots & 0 \\
0 & a_{i 2} & 0 & \cdots & 0 \\
\vdots & \vdots & \cdots & 0 & \vdots \\
0 & 0 & \cdots & a_{i\left(n_{i}-1\right)} & 0
\end{array}\right]_{n_{i} \times n_{i}}
$$


é nilpotente, digamos de ordem $s_{i}$. Desse modo, $J_{x}\left(\lambda_{i}\right)^{p}=\left(\lambda_{i} I_{n_{i}}+M_{n_{i}}\right)^{p}=\lambda_{i}^{p} I_{n_{i}}^{p}+M_{n_{i}}^{p}$. Tomando $N_{i}$ suficientemente grande a fim de que $M_{n_{i}}^{p^{N_{i}}}=0$, temos que $J_{x}\left(\lambda_{i}\right)^{p^{N_{i}}}=\lambda_{i}^{p^{N_{i}}} I_{n_{i}}^{p^{N_{i}}}$ que é uma matriz diagonal. Fazendo isso para todo $i=2, \ldots, n$, ou seja, para todos os blocos de Jordan de $x$ e tomando $\mathrm{N}$ o maior de todos os $N_{i}$ temos que $x^{p^{N}}$ é uma matriz diagonal, o que prova a afirmação. Neste caso podemos substituir $x$ por uma potência de si mesmo e assumir que $x$ é diagonal.

Defina $\mathrm{r}$ como sendo o inteiro maior ou igual a 0 tal que $r+1$ seja a dimensão do maior bloco de Jordan para um autovalor $\lambda_{i} \operatorname{com}\left|\lambda_{i}\right|=\left|\lambda_{n}\right|$ ( $\lambda_{i}$ é um autovalor minimal). Note que $r=0$ quando $\operatorname{car} K>0$, já que neste caso podemos considerar $x$ diagonal. Por outro lado, de uma maneira geral, note que dada uma matriz $x$, se $\lambda \neq 0$ for um autovalor de $x$, e $J_{x}(\lambda)$ for um bloco de Jordan de $x$ com autovalor $\lambda$, então para cada $s \in \mathbb{Z}$, a s-ésima potência de $J_{x}(\lambda)$ é da forma:

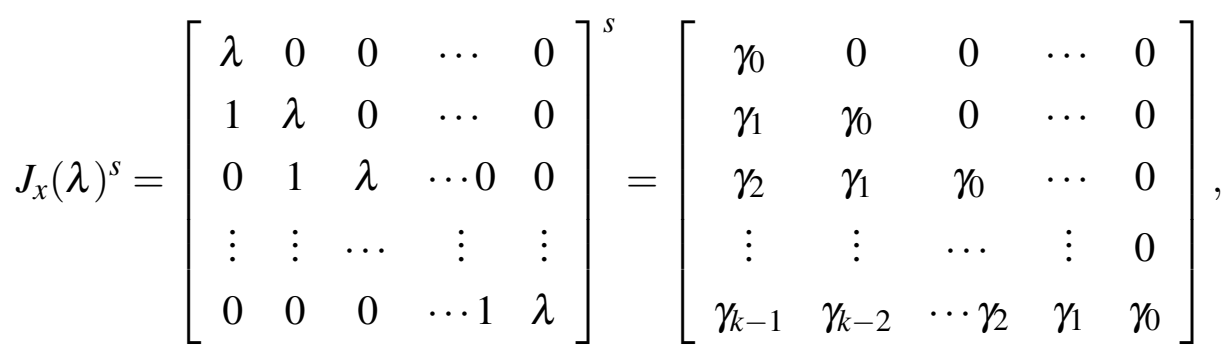

onde $\gamma_{j}=\left(\begin{array}{c}s \\ j\end{array}\right) \lambda^{s-j}, j=0,1, \ldots, k-1$.

Logo, se definirmos $\omega_{d}=d^{-r} \lambda_{n}^{d} x^{-d}$ para $d=1,2, \ldots$ então as entradas não nulas de $\omega_{d}$ têm a forma:

$$
d^{-r} \lambda_{n}^{d}\left(\begin{array}{c}
-d \\
j
\end{array}\right) \lambda_{i}^{-d-j}
$$

bastando aplicar a fórmula anterior para cada bloco de Jordan de $x$. Observe que $j \leq r$ quando $\left|\lambda_{i}\right|=\left|\lambda_{n}\right|$.

Pela escolha de $\mathrm{r}$, existe ao menos um i com $\left|\lambda_{i}\right|=\left|\lambda_{n}\right|$ onde se tenha uma entrada da forma de (2) com $j=r$, e a norma dessa entrada tende para $\frac{\left|\lambda_{n}\right|^{-r}}{r !} \neq 0$ quando $d \rightarrow \infty$, pois

$$
\begin{aligned}
& \left|d^{-r} \lambda_{n}^{d}\left(\begin{array}{c}
-d \\
r
\end{array}\right) \lambda_{i}^{-d-r}\right|=\left|d^{-r}\right| \cdot|(-d)(-d-1)(-d-2) \ldots(-d-r+1)| \cdot\left|\frac{\lambda_{n}^{-r}}{r !}\right|= \\
= & \left|\frac{d(d+1)(d+2) \ldots(d+r-1)}{d^{r}}\right| \frac{\left|\lambda_{n}^{-r}\right|}{r !}=\left|\left(1+\frac{1}{d}\right) \ldots\left(1+\frac{r-1}{d}\right)\right| \cdot \frac{\left|\lambda_{n}\right|^{-r}}{r !} \longrightarrow \frac{\left|\lambda_{n}\right|^{-r}}{r !}
\end{aligned}
$$

quando $d \longrightarrow \infty$.

Por outro lado, observe de (2) que quando $j>r$, temos $\left|\lambda_{i}\right|>\left|\lambda_{n}\right|$ e assim, para d suficientemente grande, as entradas (em norma) de $\omega_{d}$ ficam limitadas por $\left|\lambda_{n}\right|^{-r}$. De fato, vamos analisar dois casos separadamente: 
1ํㅡㄴ Caso: Se $\operatorname{car} K=p>0$. Nesse caso, como já vimos no início desta prova, podemos considerar $x$ diagonal e portanto:

$$
\begin{gathered}
\omega_{d}=d^{-r} \lambda_{n}^{d} x^{-d}=d^{-r} \lambda_{n}^{d}\left(\begin{array}{ccccc}
\lambda_{1}^{-d} & 0 & 0 & \cdots & 0 \\
0 & \lambda_{2}^{-d} & 0 & \cdots & 0 \\
0 & 0 & \cdots & & 0 \\
\vdots & \vdots & \ldots & & \vdots \\
0 & 0 & 0 & \cdots & \lambda_{n}^{-d}
\end{array}\right)= \\
=\left(\begin{array}{ccccc}
d^{-r} \lambda_{n}^{d} \lambda_{1}^{-d} & 0 & 0 & \cdots & 0 \\
0 & d^{-r} \lambda_{n}^{d} \lambda_{2}^{-d} & 0 & \cdots & 0 \\
0 & 0 & \cdots & & 0 \\
\vdots & \vdots & \cdots & & \vdots \\
0 & 0 & 0 & \cdots & d^{-r} \lambda_{n}^{d} \lambda_{n}^{-d}
\end{array}\right)
\end{gathered}
$$

$\operatorname{com}\left|d^{-r} \lambda_{n}^{d} \lambda_{i}^{-d}\right| \leq\left|d^{-r} \lambda_{n}^{d} \lambda_{1}^{-d}\right|<\left|d^{-r}\right|=1=\left|\lambda_{n}\right|^{-r}$, já que $r=0$ quando $\operatorname{car} K>0$.

$2^{\underline{0}}$ Caso: $\operatorname{Se} \operatorname{car} K=0$. Note que

$$
\left|d^{-r} \lambda_{n}^{d}\left(\begin{array}{c}
-d \\
j
\end{array}\right) \lambda_{i}^{-d-j}\right|=\left|\frac{d(d+1) \ldots(d+j-1)}{d^{r}}\right| \cdot\left(\frac{\left|\lambda_{n}\right|}{\left|\lambda_{i}\right|}\right)^{d} \cdot\left(\frac{\left|\lambda_{i}\right|^{-j}}{j !}\right) \longrightarrow 0
$$

quando $d \longrightarrow \infty$, pois $0<\frac{\left|\lambda_{n}\right|}{\left|\lambda_{i}\right|}<1$ Logo, para d suficientemente grande, as entradas de $\omega_{d}$ pertencem ao conjunto

$$
B=\left\{y \in K:|y| \leq\left|\lambda_{n}\right|^{-r}\right\}
$$

Como K é localmente compacto segue que B é compacto e pelo fato de $\mathrm{K}$ também ser completo (já que é localmente compacto) existe uma subsequência $\left\{\omega_{d_{k}}\right\}$ de $\left\{\omega_{d}\right\}$ que converge para uma matriz $\omega \neq 0$, definida sobre $\mathrm{K}$.

Considere agora o conjunto:

$$
S=\{(u, v) \in G \times G: \text { a entrada }(1,1) \text { de u } \omega \text { v é } 0\} .
$$

Claramente S é um subconjunto fechado de $G \times G$ na topologia de Zariski. Afirmamos que $\mathrm{S}$ é um subconjunto próprio. Com efeito, suponha por absurdo que $S=G \times G$. Então eu $\omega v e^{T}=0$, para todos $u, v \in G$, onde $e=\left(\begin{array}{c}1 \\ \vdots \\ 0\end{array}\right) \in K^{n}$ e $e^{T}$ é a transposta de $e$. 
Como $\omega \neq 0$, segue que existe $f \in K^{n}$ tal que $\omega f \neq 0$. Agora como por hipótese $\mathrm{G}$ é irredutível, segue pelo Lema 3.0.2 que existem combinações K-lineares $a$ e $b$ de matrizes em $\mathrm{G}$ tais que $a e=f$ e $b(\omega f)=e$. Portanto $0=e^{T}(b \omega) a e=e^{T}(b \omega f)=e e^{T}=1$, o que nos dá uma contradição. Logo $\mathrm{S}$ é de fato um subconjunto fechado próprio de $G \times G$ na topologia de Zariski. De maneira similar, definindo

$$
S^{-1}=\left\{\left(u^{-1}, v^{-1}\right) \in G \times G:(u, v) \in S\right\}
$$

temos que este também é um subconjunto fechado próprio de $G \times G$ na topologia de Zariski. Por hipótese, $\mathrm{G}$ é conexo na topologia de Zariski. Logo $G \times G$ também é conexo e pelo Teorema 1.1.1, $G \times G$ é irredutível como espaço topológico, ou seja, $G \times G$ não pode ser a união de dois subconjuntos fechados próprios. Em outras palavras, existem $y, z \in G$ tais que $\left(y^{-1}, z\right)$ não pertence a $S \cup S^{-1}$, e ambas matrizes $y^{-1} \omega z$ e $y \omega z^{-1}$ possuem suas entradas de posição $(1,1)$, digamos $\mu_{1} \mathrm{e}$ $\mu_{2}$, respectivamente, não nulas.

Agora defina:

$$
f_{d}=d^{-r} \lambda_{n}^{d} \lambda_{1}^{-d} x^{d} y^{-1} x^{-d} z=\left(\lambda_{1}^{-d} x^{d}\right) y^{-1} \omega_{d} z
$$

$\mathrm{e}$

$$
g_{d}=d^{-r} \lambda_{n}^{d} \lambda_{1}^{-d} x^{d} y x^{-d} z^{-1}=\left(\lambda_{1}^{-d} x^{d}\right) y \omega_{d} z^{-1}
$$

Afirmação: $\lambda_{1}^{-d} x^{d} \longrightarrow\left[\begin{array}{cccc}1 & 0 & \cdots & 0 \\ 0 & 0 & \cdots & 0 \\ \vdots & \vdots & \vdots & \vdots \\ 0 & 0 & \cdots & 0\end{array}\right]$ quando $d \longrightarrow \infty$. De fato, novamente analizemos os casos em que $\operatorname{car} K=p>0$ e quando $\operatorname{car} K=0$ separadamente.

Se $\operatorname{car} K=p>0$, então como podemos considerar neste caso que $x$ é uma matriz diagonal, segue que $x^{d}$ é da forma :

$$
x^{d}=\left[\begin{array}{cccc}
\lambda_{1}^{d} & 0 & \cdots & 0 \\
0 & \lambda_{2}^{d} & \cdots & 0 \\
\vdots & \vdots & \vdots & \vdots \\
0 & 0 & \cdots & \lambda_{n}^{d}
\end{array}\right]
$$

e daí,

$$
\lambda_{1}^{-d} x^{d}=\left[\begin{array}{cccc}
1 & 0 & \cdots & 0 \\
0 & \left(\frac{\lambda_{2}}{\lambda_{1}}\right)^{d} & \cdots & 0 \\
\vdots & \vdots & \vdots & \vdots \\
0 & 0 & \cdots & \left(\frac{\lambda_{n}}{\lambda_{1}}\right)^{d}
\end{array}\right] \longrightarrow\left[\begin{array}{cccc}
1 & 0 & \cdots & 0 \\
0 & 0 & \cdots & 0 \\
\vdots & \vdots & \vdots & \vdots \\
0 & 0 & \cdots & 0
\end{array}\right]
$$


quando $d \longrightarrow \infty$, pois como $\lambda_{1}$ é o autovalor dominante de $x$.

Se $c a r K=0$, da mesma forma que (2), as entradas não nulas de $\lambda_{1}^{-d} x^{d}$ são do tipo:

$$
\lambda_{1}^{-d}\left(\begin{array}{c}
d \\
j
\end{array}\right) \lambda_{i}^{d-j}=\underbrace{\frac{d !}{(d-j) !}}_{\text {função pol. exp. decresc. }} \cdot \underbrace{\left(\frac{\lambda_{i}}{\lambda_{1}}\right)^{d}}_{\text {. }} \cdot \frac{\lambda_{i}^{-j}}{j !} \longrightarrow 0
$$

quando $d \longrightarrow \infty, i=2, \ldots, n$. Observe ainda que a entrada $(1,1)$ de $\lambda_{1}^{-d} x^{d}$ é igual a 1 . Assim,

$$
\lambda_{1}^{-d} x^{d} \longrightarrow\left[\begin{array}{cccc}
1 & 0 & \cdots & 0 \\
0 & 0 & \cdots & 0 \\
\vdots & \vdots & \vdots & \vdots \\
0 & 0 & \cdots & 0
\end{array}\right]
$$

o que prova a afirmação.

Portanto, como $\left\{\omega_{d_{k}}\right\}$ converge para $\omega$, concluimos que $\left\{f_{d_{k}}\right\}$ e $\left\{g_{d_{k}}\right\}$ convergem para matrizes da forma

$$
\left[\begin{array}{cccc}
\mu_{1} & * & \cdots & * \\
0 & 0 & \cdots & 0 \\
\vdots & \vdots & \vdots & \vdots \\
0 & 0 & \cdots & 0
\end{array}\right] e\left[\begin{array}{cccc}
\mu_{2} & * & \cdots & * \\
0 & 0 & \cdots & 0 \\
\vdots & \vdots & \vdots & \vdots \\
0 & 0 & \cdots & 0
\end{array}\right],
$$

respectivamente.

Portanto, para uma infinidade de inteiros $\mathrm{d}, f_{d}$ e $g_{d}$ terão ambos autovalores dominantes (aproximadamente $\mu_{1}$ e $\mu_{2}$, respectivamente). Escolhendo um desses valores de d e definindo $x_{0}=x^{d} y^{-1} x^{-d} z \in G$, temos que $x_{0}=\beta f_{d}$, onde $\beta=d^{r} \lambda_{n}^{-d} \lambda_{1}^{d} \in K$ e $x_{0}^{-1}=\beta z^{-1} g_{d} z$. Note que $|\beta|=d^{r}$. $\left|\frac{\lambda_{1}}{\lambda_{n}}\right|^{d}>1$. Logo $x_{0}$ tem autovalor dominante aproximadamente $\beta \mu_{1}$ e $x_{0}^{-1}$ terá autovalor dominante aproximadamente $\beta \mu_{2}$, já que $z^{-1} g_{d} z$ é semelhante à $g_{d}$ e matrizes semelhantes possuem os mesmos autovalores.

\section{Resumo dos passos anteriores:}

Vamos agora resumir o que conseguimos nos 6 passos anteriores e preparar para o passo final que concluirá a demonstração do teorema de Alternativa de Tits.

Estamos supondo que $G \leq G L(n, F)$ é um grupo linear finitamente gerado e que $G$ não é solúvel por finito. Queremos mostrar que $G$ contém um subgrupo livre a dois geradores. Pelos passos $1 \mathrm{e}$ 2 podemos assumir que $G$ é completamente redutível e conexo na topologia de Zariski. 
Usando a hipótese que $G$ não é solúvel por finito e é completamente redutível, no passo 3 exibimos $x \in G^{\prime}$ que possui um autovalor $\xi$ que não é uma raiz da unidade. O passo 4 consiste em estender o corpo de base $F$ a um corpo $(K,||)$ localmente compacto satisfazendo $|\xi| \neq 1$. Naturalmente podemos considerar $G \leq G L(n, K)$.

O passo 5 nos dá uma nova representação de $G, \rho: G \longrightarrow G L(m, K)$ tal que $\rho(x)$ tem um autovalor dominante. Considere a imagem homomórfica $\rho(G) \leq G L(m, K)$. Como $G$ é conexo segue-se que $\rho(G)$ também é conexo. No entanto, para aplicar o passo 6, precisamos que $\rho(G)$ seja também irredutível o que não é necessariamente verdade, uma vez que mudamos de representação.

Para sanar esse problema voltamos a aplicar uma variante do passo 1: considere uma sequência de composição de $\rho(G), V=V_{0} \supset V_{1} \supset \cdots \supset V_{t}=\{0\}$. Numa base associada a essa decomposição, $\rho(x)$ se escreve como uma matriz triangular, digamos:

$$
\left(\begin{array}{cccc}
x_{1} & & & \\
& x_{2} & & 0 \\
* & & \ddots & \\
& & & x_{h}
\end{array}\right)
$$

O autovalor dominante de $\rho(x)$ será então um autovalor dominante de $\rho_{i}(x)$ para alguma representação irredutível

$$
\begin{gathered}
\rho_{i}: \rho(G) \longrightarrow G L\left(\frac{V_{i-1}}{V_{i}}\right) \\
\rho(x) \longmapsto \rho_{i}(x)
\end{gathered}
$$

Agora a imagem homomórfica de $G, \rho_{i}(\rho(G)) \leq G L\left(\frac{V_{i-1}}{V_{i}}\right)$ é um grupo linear conexo e irredutível que contém um elemento $\rho_{i}(x)$ com um autovalor dominante. É importante notar, para o próximo passo, que o grau da representação $\rho_{i}$ é maior que 1 . De fato, como $x \in G^{\prime}$ então $\rho_{i}(x) \in\left(G L\left(\frac{V_{i-1}}{V_{i}}\right)\right)^{\prime}$ e é não trivial.

Trocando, mais uma vez, $\rho_{i}(\rho(G)) \leq G L\left(\frac{V_{i-1}}{V_{i}}\right)$ por $G \leq G L(n, K)$ e $\rho_{i}(x) \in \rho_{i}(\rho(G))$ por $x \in G$, temos que $G$ é conexo e irredutível e $x$ tem um autovalor dominante.

Podemos agora aplicar o passo 6 para garantir a existência de $x_{0} \in G$ tal que ambos $x_{0}$ e $x_{0}^{-1}$ têm autovalores dominantes.

\section{Passo 7 - Existência de um grupo livre de posto 2 em $G$}

O próximo Teorema, precedido de três Lemas auxiliares, nos garantirá a existência de um grupo livre de posto $2 \mathrm{em} G$. 
Lema 3.0.4 Seja $F$ um corpo e $G \leq G L(n, F)$. Sejam ainda $V_{1}$ e $V_{2}$ subespaços não nulos de $F^{n}$. Então o conjunto

$$
L\left(V_{1}, V_{2}\right):=\left\{z \in G: z V_{1} \cap V_{2} \neq\{0\}\right\}
$$

é um fechado de Zariski em $G$. Além disso, se $G$ for irredutível e $\operatorname{dim}\left(V_{1}\right)=\operatorname{dim}\left(V_{2}\right)=1$, então $L\left(V_{1}, V_{1}\right)$ e $L\left(V_{2}, V_{2}\right)$ são subconjuntos próprios de $G$.

Demonstração: Observe primeiro que $z V_{1} \cap V_{2} \neq\{0\}$ se e somente se $\operatorname{dim}\left(z V_{1}+V_{2}\right)<\operatorname{dim}\left(V_{1}\right)+$ $\operatorname{dim}\left(V_{2}\right)$, pois pela fórmula da dimensão temos:

$$
\operatorname{dim}\left(z V_{1}+V_{2}\right)=\operatorname{dim}\left(z V_{1}\right)+\operatorname{dim}\left(V_{2}\right)-\operatorname{dim}\left(z V_{1} \cap V_{2}\right)
$$

Mas $\operatorname{dim}\left(z V_{1}\right)=\operatorname{dim}\left(V_{1}\right)$. Assim,

$$
\operatorname{dim}\left(z V_{1}+V_{2}\right)=\operatorname{dim}\left(V_{1}\right)+\operatorname{dim}\left(V_{2}\right)-\operatorname{dim}\left(z V_{1} \cap V_{2}\right)
$$

Agora seja $z=\left(z_{i j}\right)_{n \times n} \in G$ e considere $B_{V_{1}}=\left\{v_{1}, \ldots, v_{s}\right\}$ e $B_{V_{2}}=\left\{w_{1}, \ldots, w_{t}\right\}$ bases de $V_{1}$ e $V_{2}$, respectivamente. Logo, $B_{z V_{1}}=\left\{z v_{1}, \ldots, z v_{s}\right\}$ é uma base para $z V_{1}$. Note que para todo $v_{i}=\left(v_{i 1}, v_{i 2}, \ldots, v_{i n}\right)$ e $w_{j}=\left(w_{j 1}, w_{j 2}, \ldots, w_{j n}\right), 1 \leq i \leq s, 1 \leq j \leq t$ tem-se:

$$
\begin{gathered}
z v_{i}=\left(\begin{array}{ccc}
z_{11} & \cdots & z_{1 n} \\
\vdots & \ddots & \vdots \\
z_{n 1} & \cdots & z_{n n}
\end{array}\right)\left(\begin{array}{c}
v_{i 1} \\
\vdots \\
v_{i n}
\end{array}\right)=\left(\begin{array}{c}
z_{11} v_{i 1}+z_{12} v_{i 2}+\cdots+z_{1 n} v_{i n} \\
\vdots \\
z_{n 1} v_{i 1}+z_{n 2} v_{i 2}+\cdots+z_{n n} v_{i n}
\end{array}\right) e \\
z w_{j}=\left(\begin{array}{ccc}
z_{11} & \cdots & z_{1 n} \\
\vdots & \ddots & \vdots \\
z_{n 1} & \cdots & z_{n n}
\end{array}\right)\left(\begin{array}{c}
w_{j 1} \\
\vdots \\
w_{j n}
\end{array}\right)=\left(\begin{array}{c}
z_{11} w_{j 1}+\cdots+z_{1 n} w_{j n} \\
\vdots \\
z_{n 1} w_{j 1}+\cdots+z_{n n} w_{j n}
\end{array}\right) .
\end{gathered}
$$

Logo, $V_{1} z+V_{2}$ é gerado pelas linhas da seguinte matriz $M$ :

$$
M=\left(\begin{array}{cccc}
\left(z v_{1}\right)_{1} & \left(z v_{1}\right)_{2} & \cdots & \left(z v_{1}\right)_{n} \\
\left(z v_{2}\right)_{1} & \left(z v_{2}\right)_{2} & \cdots & \left(z v_{2}\right)_{n} \\
\vdots & \vdots & \ddots & \vdots \\
\left(z v_{s}\right)_{1} & \left(z v_{s}\right)_{2} & \cdots & \left(z v_{s}\right)_{n} \\
w_{11} & w_{12} & \cdots & w_{1 n} \\
w_{21} & w_{22} & \cdots & w_{2 n} \\
\vdots & \vdots & \ddots & \vdots \\
w_{t 1} & w_{t 2} & \cdots & w_{t n}
\end{array}\right)_{(s+t) \times n}
$$


onde $\left(z v_{i}\right)_{l}=\sum_{j=1}^{n} z_{l j} v_{i j}, 1 \leq i \leq s, 1 \leq l \leq n$.

Mas $\operatorname{dim}\left(z V_{1}+V_{2}\right)=$ posto de $M$ e portanto $z V_{1} \cap V_{2} \neq\{0\}$ se, e somente se, posto de $M<$ $\underbrace{\operatorname{dim}\left(V_{1}\right)+\operatorname{dim}\left(V_{2}\right)}_{d}$. Isto ocorre se, e somente se, todos os determinantes menores de $M$ de tamanho $d$ forem nulos, ou seja, as entradas de $z$ satisfazem um conjunto de polinômios. Portanto $L\left(V_{1}, V_{2}\right)$ é um fechado de Zariski em G.

Agora suponha $\mathrm{G}$ irredutível. Temos:

$$
L\left(V_{1}, V_{1}\right)=\left\{z \in G: z V_{1} \cap V_{1} \neq\{0\}\right\}
$$

Mas se $\{0\} \neq z V_{1} \cap V_{1}$, então como $z V_{1} \cap V_{1} \subset V_{1}$ e $\operatorname{dim}\left(V_{1}\right)=\operatorname{dim}\left(z V_{1}\right)=1$, segue que $z V_{1}=V_{1}$ e daí

$$
L\left(V_{1}, V_{1}\right)=\left\{z \in G: z V_{1}=V_{1}\right\}
$$

Logo, se $L\left(V_{1}, V_{1}\right)=G$ então para todo $z \in G$, tem-se $z V_{1}=V_{1}$ e portanto $V_{1}$ seria G-invariante, o que é um absurdo pois estamos considerando $G$ irredutível. Analogamente para $L\left(V_{2}, V_{2}\right)$.

Lema 3.0.5 Seja $K$ um espaço vetorial normado localmente compacto e $A \subset K$ um subconjunto fechado. Seja ainda $S=\left\{k_{1}, \ldots, k_{t}\right\}$ um subconjunto qualquer finito de $K$, tal que $k_{i} \notin A$, para todo $i=1, \ldots, t$. Então existe uma vizinhança compacta $N_{0}$ de $S \operatorname{com} N_{0} \cap A=\emptyset$.

Demonstração: Como por hipótese $A$ é fechado, segue que $A^{c}$ é aberto. Logo, existem abertos $B_{1}, B_{2}, \ldots, B_{t}$ tais que $k_{i} \in B_{i}$ e $B_{i} \subset A^{c}, i=1, \ldots, t$. Assim, da hipótese de $\mathrm{K}$ ser localmente compacto, existem compactos $K_{1}, \ldots, K_{t}$, tais que $k_{i} \in K_{i} \subset B_{i}, i=1,2, \ldots, t$. Note que $K_{i} \cap A=\emptyset$, $i=1, \ldots, t$. Desse modo, tomando $N_{0}=\bigcup_{i=1}^{t} K_{i}$, temos que $N_{0}$ é compacto, $S \subset N_{0}$ e $N_{0} \cap A=\emptyset$, como queríamos.

Lema 3.0.6 Seja $x \in G L(n, K)$ com um autovalor dominante $\lambda$. Seja ainda $\alpha \neq \lambda$ um outro autovalor de $x$ e $J_{x}(\alpha)$ um bloco de Jordan de $\alpha$, com base $f_{1}, f_{2}, \ldots, f_{t}$. Então a sequência $\lambda^{-k} x^{k} f_{i} \longrightarrow 0$, quando $k \longrightarrow \infty$, para todo $i=1,2, \ldots, t$.

Demonstração: Seja $J_{x}(\alpha)=\left(\begin{array}{ccccc}\alpha & 1 & 0 & \cdots & 0 \\ 0 & \alpha & 1 & \cdots & 0 \\ 0 & 0 & \alpha & \ddots & \vdots \\ \vdots & \vdots & \vdots & \ddots & 1 \\ 0 & 0 & 0 & \cdots & \alpha\end{array}\right)_{t \times t}$. Assim, 
$x f_{1}=\alpha f_{1}$

$x f_{2}=\alpha f_{2}+f_{1}$

:

$x f_{t}=\alpha f_{t}+f_{t-1}$. Vamos definir $f_{i}=0$ se $i \leq 0$. Assim,

$$
x^{2} f_{i}=\alpha^{2} f_{i}+2 \alpha f_{i-1}+f_{i-2}=\left(\begin{array}{l}
2 \\
0
\end{array}\right) \alpha^{2} f_{i}+\left(\begin{array}{l}
2 \\
1
\end{array}\right) \alpha^{2-1} f_{i-1}+\left(\begin{array}{l}
2 \\
2
\end{array}\right) \alpha^{2-2} f_{i-2}
$$

De maneira geral, dado $k \geq 1$,

$x^{k} f_{i}=\left(\begin{array}{c}k \\ 0\end{array}\right) \alpha^{k} f_{i}+\left(\begin{array}{c}k \\ 1\end{array}\right) \alpha^{k-1} f_{i-1}+\left(\begin{array}{c}k \\ 2\end{array}\right) \alpha^{k-2} f_{i-2}+\cdots+\left(\begin{array}{c}k \\ k-1\end{array}\right) \alpha f_{i-k+1}+\left(\begin{array}{c}k \\ k\end{array}\right) f_{i-k}$

Desse modo,

$$
\begin{gathered}
\lambda^{-k} x^{k} f_{i}=\left(\begin{array}{c}
k \\
0
\end{array}\right) \lambda^{-k} \alpha^{k} f_{i}+\lambda^{-1}\left(\begin{array}{c}
k \\
1
\end{array}\right) \lambda^{-(k-1)} \alpha^{k-1} f_{i-1}+\cdots+\lambda^{-k}\left(\begin{array}{l}
k \\
k
\end{array}\right) f_{i-k}= \\
\sum_{j=0}^{k}\left(\frac{\alpha}{\lambda}\right)^{k-j} \lambda^{-j}\left(\begin{array}{c}
k \\
j
\end{array}\right) f_{i-j} . \quad(*)
\end{gathered}
$$

Mas note que como $|\alpha|<|\lambda|$, segue que $\left|\frac{\alpha}{\lambda}\right|<1$. Ainda, $\left(\begin{array}{c}k \\ j\end{array}\right)=\frac{k(k-1) \ldots(k-j+1)}{j !}$. Logo, $\left|\left(\begin{array}{c}k \\ j\end{array}\right)\right| \cdot\left|\frac{\alpha}{\lambda}\right|^{k} \longrightarrow 0$, quando $k \longrightarrow \infty . \quad(* *)$

De $(*)$ e $(* *)$ temos:

$$
\begin{gathered}
\left|\lambda^{-k} x^{k} f_{i}\right|=\left|\sum_{j=0}^{k} \lambda^{-j}\left(\frac{\alpha}{\lambda}\right)^{k-j}\left(\begin{array}{c}
k \\
j
\end{array}\right) f_{i-j}\right| \leq \sum_{j=0}^{k}\left|\lambda^{-j}\right|\left|\frac{\alpha}{\lambda}\right|^{k-j}\left|\left(\begin{array}{c}
k \\
j
\end{array}\right)\right|\left|f_{i-j}\right|= \\
=\sum_{j=0}^{k}\left|\alpha^{-j}\right|\left|\frac{\alpha}{\lambda}\right|^{k}\left|\left(\begin{array}{c}
k \\
j
\end{array}\right)\right|\left|f_{i-j}\right| \longrightarrow 0
\end{gathered}
$$

quando $k \longrightarrow \infty$ e portanto $\lambda^{-k} x^{k} f_{i} \longrightarrow 0$ quando $k \longrightarrow \infty$, para todo $i=1, \ldots, t$.

Teorema 3.0.3 Seja $G \leq G L(n, K)$ um grupo linear irredutivel de grau $n>1$ o qual é conexo na topologia de Zariski. Suponha que G possua um elemento $x$ tal que ambos $x$ e $x^{-1}$ tenham autovalores dominantes. Então, para algum conjugado $y=b^{-1} x b$ de $x$ em $G$, existe um inteiro $k \geq 1$ tal que $\left\langle x^{k}, y^{k}\right\rangle$ é um subgrupo livre de posto 2 . 
Demonstração: Sejam $\lambda$ e $\mu$ os autovalores dominantes de $x$ e $x^{-1}$, respectivamente e sejam $u_{1} \mathrm{e}$ $u_{2}$ seus autovetores correspondentes. Então $x u_{1}=\lambda u_{1}$ e $x u_{2}=\mu^{-1} u_{2}$.

Tome $U_{1}=K u_{1}, U_{2}=K u_{2}$ e seja $U_{3}$ o subespaço $x$-invariante tal que $K^{n}=U_{1} \oplus U_{2} \oplus U_{3}$, o qual existe pelo fato de $\mathrm{G}$ ser completamente redutível. Para simplificar a notação, escrevemos $U_{1}$ ' $=U_{2} \oplus U_{3}, U_{2}{ }^{\prime}=U_{1} \oplus U_{3}$ e $U_{3}{ }^{\prime}=U_{1} \oplus U_{2}$. Vamos utilizar o Lema do Ping-Pong para provarmos esta proposição. Para isso, consideremos os seguintes subgrupos de G:

$$
A=\left\langle x^{k}\right\rangle \text { e } B=\left\langle b x^{k} b^{-1}\right\rangle, \text { para algum } \mathrm{k} \text {, a ser definido. }
$$

Nosso próximo passo é achar os conjuntos $\Gamma$ e $\Delta$ satisfazendo as condições do Lema do PingPong, relacionando-os aos subgrupos A e B considerados anteriormente. Antes disso, observe que independentemente de sabermos ou não quais conjuntos iremos considerar como tais $\Gamma$ e $\Delta$, se estes satisfizerem a relação $\Gamma=b \Delta$, então as hipóteses do Lema do Ping-Pong se resumem a:

1. $\Delta \cap b \Delta=\emptyset$

2. $x^{k r}\left(b \Delta \cup b^{-1} \Delta\right) \subseteq \Delta$, para todo inteiro $r \neq 0$

3. $x^{k r} \Delta \cap \Delta \neq \emptyset$, para todo inteiro $r \neq 0$.

Começamos por achar $b$. Observe que pelo Lema 3.0.3, os conjuntos $L\left(U_{i}, U_{i}^{\prime}\right), L\left(U_{i}^{\prime}, U_{i}\right)$ e $L\left(U_{i}, U_{i}\right), i=1,2$, são subconjuntos fechados de $\mathrm{G}$. Ainda, pelo mesmo resultado, $L\left(U_{i}, U_{i}\right), i=1,2$ são subconjuntos próprios de $\mathrm{G}$. Note que $L\left(U_{i}, U_{i}^{\prime}\right)$ e $L\left(U_{i}^{\prime}, U_{i}\right), i=1,2$, também são subconjuntos próprios de G. De fato, mostremos para $L\left(U_{1}, U_{1}^{\prime}\right)$ pois os outros conjuntos seguem de maneira análoga. Temos:

$$
L\left(U_{1}, U_{1}^{\prime}\right)=L\left(U_{1}, U_{2} \oplus U_{3}\right)=\left\{z \in G: z U_{1} \cap\left(U_{2} \oplus U_{3}\right) \neq\{0\}\right\} .
$$

Note que $1 \in G$ mas $1 \notin L\left(U_{1}, U_{1}^{\prime}\right)$, pois se $1 \in L\left(U_{1}, U_{1}^{\prime}\right)$ então $U_{1} \cap\left(U_{2} \oplus U_{3}\right) \neq\{0\}$, o que é um absurdo já que $K^{n}=U_{1} \oplus U_{2} \oplus U_{3}$.

Agora como por hipótese G é conexo na topologia de Zariski, segue pelo Teorema 1.1.1 que G não pode ser a união de um número finito de subconjuntos fechados próprios. Desse modo, existe $b \in G$ o qual não pertence a nenhum dos conjuntos:

$$
L\left(U_{i}, U_{i}^{\prime}\right), L\left(U_{i}^{\prime}, U_{i}\right) \text { e } L\left(U_{i}, U_{i}\right), i=1,2
$$

Equivalentemente, temos $b U_{i} \cap U_{i}^{\prime}=\{0\}, b U_{i} \cap U_{i}=\{0\}$ e $b U_{i}^{\prime} \cap U_{i}=\{0\}$, onde este último implica que $U_{i}^{\prime} \cap b^{-1} U_{i}=\{0\}$. 
Em consequência disso, segue também que:

$$
\left(b U_{i} \cup b^{-1} U_{i}\right) \cap\left(U_{i} \cup U_{i}^{\prime}\right)=\{0\}, i=1,2
$$

Vamos construir $\Delta$. Como K é um corpo normado localmente compacto, segue que podemos definir uma norma || em $K^{n}$, por exemplo $\left|\left(\alpha_{1}, \alpha_{2}, \ldots, \alpha_{n}\right)\right|:=\max _{1 \leq i \leq n}\left|\alpha_{i}\right|$, de modo a tornar $\left(K^{n},||\right)$ um espaço vetorial localmente compacto.

Por (1), vem que o conjunto $\left\{u_{1}, u_{2}\right\}$ é disjunto de $S:=U_{3} \cup b U_{1}^{\prime} \cup b^{-1} U_{1}^{\prime} \cup b U_{2}^{\prime} \cup b^{-1} U_{2}^{\prime}$. Por outro lado, ( vide Proposição 12 de [9], pg. 238 ) segue que todo subespaço de $K^{n}$ é fechado na topologia da norma. Assim, pelo Lema 3.0.4, existe uma vizinhança compacta $N_{0}$ de $\left\{u_{1}, u_{2}\right\}$ com $N_{0} \cap S=\emptyset$.

Ponha $W_{0}:=\left\{\alpha u: \alpha \in K, u \in N_{0}\right\}$. Daí, $W_{0} \cap S=\{0\}$. Note que $W_{0}$ é fechado na topologia da norma. De fato, seja $\left(\alpha_{i} w_{i}\right)$ sequência em $W_{0}$ convergindo para um ponto $v=\left(v_{1}, \ldots, v_{n}\right)$, $\alpha_{i} \in K, w_{i} \in N_{0}$. Como $N_{0}$ é compacto, podemos assumir (restringindo a uma subsequência se necessário ) que $\left(w_{i}\right)$ converge para algum ponto $w \in N_{0}$. Observe que $w \neq 0$, pois $0 \notin N_{0}$, já que $0 \in S$ e $S \cap N_{0}=\emptyset$. Logo, podemos considerar que uma componente de $w=\left(w_{1}, \ldots, w_{n}\right)$ é não nula, a qual sem perda de generalidade, podemos supor que seja $w_{1}$. Ainda, se $w_{i}=\left(w_{1}^{i}, w_{2}^{i}, \ldots, w_{n}^{i}\right) \in K^{n}$, vem que $w_{1}^{i} \longrightarrow w_{1}$, quando $i \longrightarrow \infty$. Por outro lado, como $\left(\alpha_{i} w_{i}\right) \longrightarrow v=\left(v_{1}, \ldots, v_{n}\right)$, segue que $\left(\alpha_{i} w_{1}^{i}\right) \longrightarrow v_{1}$ e como $w_{1} \neq 0$, segue que para $m \in \mathbb{N}$ suficientemente grande, $w_{1}^{i} \neq 0$, para todo $i \geq m$. Assim, existe $\left(w_{1}^{i}\right)^{-1}$, para todo $i \geq m$ e podemos escrever $\alpha_{i}=\left(\alpha_{i} w_{1}^{i}\right)\left(w_{1}^{i}\right)^{-1}$, para todo $i \geq m$. Mas $\left(\alpha_{i} w_{1}^{i}\right) \longrightarrow v_{1} \mathrm{e}\left(w_{1}^{-i}\right) \longrightarrow w_{1}^{-1}$ (pois $\left.\left(w_{1}^{i}\right) \longrightarrow w_{1}\right)$. Logo, $\left(\alpha_{i}\right) \longrightarrow v_{1} w_{1}^{-1}=\alpha \in K$. Consequentemente $v=\alpha w \in W_{0}$, o que mostra que $W_{0}$ é fechado.

Observe que $W_{0} \cap\left\{b u_{1}, b u_{2}\right\}=\emptyset$, pois $W_{0} \cap\left\{b u_{1}, b u_{2}\right\} \subseteq W_{0} \cap S=\{0\}$, e $0 \notin\left\{b u_{1}, b u_{2}\right\}$. Desse modo, pelo Lema 3.0.4, podemos encontrar uma vizinhança compacta $N$ de $\left\{u_{1}, u_{2}\right\}$ tal que $N \subseteq N_{0}$ e $W_{0} \cap b N=\emptyset$.

Finalmente, tomemos:

$$
\Delta:=\{\alpha u: \alpha \in K, u \in N \text { e } \alpha \neq 0\} \text { e } \Gamma=\Delta b
$$

Como $\Delta \subseteq W_{0}$, segue que $\Delta \cap b \Delta=\emptyset$, e assim nossa condição 1 do Lema do Ping-Pong é satisfeita. Agora observe que $u_{1} \in \Delta$, pois $u_{1}=\underbrace{1}_{\in K} \cdot \underbrace{u_{1}}_{\in N}$. Ainda, $u_{1} \in x^{k r} \Delta, \forall r \in \mathbb{N}^{*}$, pois

$$
u_{1}=x^{k r}\left((\underbrace{\left.\lambda^{k r}\right)^{-1} u_{1}}_{\in \Delta})\right.
$$

já que $\lambda$ é autovalor de $x$. Assim, nossa condição 3 do Lema do Ping-Pong também é satisfeita. Agora, resta escolhermos $k \geq 1$ a fim de que nossa condição 2 do Lema do Ping-Pong seja satisfeita. Primeiro note que como $U_{1}$ é o autoespaço para o autovalor dominante $\lambda$ de $x$, segue que $\left(\lambda^{-k} x^{k} u\right) \longrightarrow$ 
0 quando $k \longrightarrow \infty$, para todo $u \in U_{1}^{\prime}$. De fato, seja $u \in U_{1}^{\prime}$. Então $u=\alpha_{2} u_{2}+u_{3}, \alpha_{2} \in K, u_{2} \in U_{2} \mathrm{e}$ $u_{3} \in U_{3}$. Daí,

$$
\lambda^{-k} x^{k} u=\lambda^{-k} x^{k}\left(\alpha_{2} u_{2}+u_{3}\right)=\left(\lambda^{-k} \alpha_{2}\right)\left(x^{k} u_{2}\right)+\lambda^{-k}\left(x^{k} u_{3}\right)=\alpha_{2} \lambda^{-k} \mu^{-k} u_{2}+\lambda^{-k} x^{k} u_{3} .
$$

Sendo também $\mu^{-1}$ autovalor de $x$, então $\left|\mu^{-1}\right|<|\lambda|$, o que implica que $\frac{\left|\mu^{-1}\right|}{|\lambda|}=\left|\frac{1}{\lambda \mu}\right|<1$. Assim, $\left|\frac{1}{\lambda \mu}\right|^{k} \longrightarrow 0$ quando $k \longrightarrow \infty$ e portanto $\lambda^{-k} \mu^{-k}=\frac{1}{\lambda \mu} \longrightarrow 0$, quando $k \longrightarrow \infty$.

Desse modo, temos $\alpha_{2} \lambda^{-k} \mu^{-k} u_{2} \longrightarrow 0$ quando $k \longrightarrow \infty$ e pelo Lema 3.0.6 vem que $\lambda^{-k} x^{k} u_{3} \longrightarrow 0$ quando $k \longrightarrow \infty$. Portanto $\lambda^{-k} x^{k} u \longrightarrow 0$, quando $k \longrightarrow \infty$, para todo $u \in U_{1}^{\prime}$.

De maneira análoga, verifica-se que $\mu^{-k} x^{k} u \longrightarrow 0$ quando $k \longrightarrow \infty$, para todo $u \in U_{2}^{\prime}$. Diante disso, se $v \in K^{n}$ for escrito da forma

$$
v=\alpha_{1} u_{1}+\alpha_{2} u_{2}+u_{3}, \operatorname{com} \alpha_{1}, \alpha_{2} \in K \text { e } u_{3} \in U_{3},
$$

então $\lambda^{-k} x^{k} v \longrightarrow \alpha_{1} u_{1}$ e $\mu^{-k} x^{-k} v \longrightarrow \alpha_{2} u_{2}$, quando $k \longrightarrow \infty$.

Observe agora que como $N \cap S=\emptyset$, segue que:

$$
\left(b N \cup b^{-1} N\right) \cap\left(U_{1}^{\prime} \cup U_{2}^{\prime}\right)=\emptyset .
$$

Ainda, $\Delta$ é uma vizinhança de $\alpha_{1} u_{1}$ e $\alpha_{2} u_{2}$ quando $\alpha_{1}$ e $\alpha_{2}$ forem não nulos, e $b N \cup b^{-1} N$ é um compacto.

Logo, existe um inteiro $k \geq 1$ tal que $l \geq k$ implica que $\lambda^{-l} x^{l} v \in \Delta$ e $\mu^{-l} x^{l} v \in \Delta$, para todo $v \in$ $b N \cup b^{-1} N$. Desse modo:

$$
x^{k r}\left(b \Delta \cup b^{-1} \Delta\right) \subseteq \Delta, \text { para todo inteiro } r \neq 0,
$$

o que mostra que a nossa condição 3 do Lema do Ping-Pong é satisfeita.

Portanto, as hipóteses do Lema do Ping-Pong estão todas satisfeitas para estas escolhas de $b, \Delta, \Gamma=$ $b \Delta, k, A=\left\langle x^{k}\right\rangle$ e $B=\left\langle b x^{k} b^{-1}\right\rangle$, e assim $\left\langle x^{k}, b x^{k} b^{-1}\right\rangle$ é um subgrupo livre de posto 2, como queríamos. 


\section{Referências Bibliográficas}

[1] J. W. S. Cassels and A Frohlich (eds.), Algebraic Number Theory, Academic Press, London, 1967.

[2] J. D. Dixon, The Structure of Linear Groups, Van Nostrand-Reinhold, London, 1971.

[3] S. Lang, Algebra, Addison-Wesley, Reading, Mass., 1965.

[4] J. Tits, Free subgroups in linear groups, J. Algebra 20 (1972), 250 - 270.

[5] B. A. F. Wehrfritz, Infinite Linear Groups, Springer-Verlag, Berlin, 1973.

[6] F. Q. Gouvêa, p-adic Numbers, Second Edition, Springer - 1997.

[7] N. Jacobson, Basic Algebra II, W. H. Freeman and Company, San Francisco - 1910.

[8] C. G. Moreira, E. Tengan, F. B. Martinez e Nicolau Saldanha Teoria dos números - um passeio com primos e outros números familiares pelo mundo inteiro, Projeto Euclides, IMPA - 2010.

[9] E. L. Lima, Espaços métricos, Rio de Janeiro - IMPA - 2007. 